\title{
Organocatalytic Asymmetric Deconjugative Michael Additions
}

\author{
Mark Bell Kim Frisch and Karl Anker Jørgensen* \\ Danish National Research Foundation: Center for Catalysis, Department of \\ Chemistry, Aarhus University, DK-8000 Aarhus C, Denmark \\ kaj@chem.au.dk
}

\section{Supporting Information}

\section{Contents}

$\begin{array}{ll}\text { General Methods } & \text { S2 }\end{array}$

$\begin{array}{ll}\text { Materials } & \text { S2 }\end{array}$

Procedures and characterization data for the derivatives of the catalytic product $\mathrm{S} 3$

Procedure and characterization data for the reductive amination product 7a-j $\quad$ S9

${ }^{1} \mathrm{H}$ and ${ }^{13} \mathrm{C}$ NMR Spectra of Compounds 6a, 7a-j, 8, 9, 10, 11, 12 and $13 \quad$ S15

$\begin{array}{ll}\text { References } & \text { S32 }\end{array}$ 
General Methods. NMR spectra were acquired running at 400 and $100 \mathrm{MHz}$ for ${ }^{1} \mathrm{H}$ and ${ }^{13} \mathrm{C}$, respectively. Chemical shifts $(\delta)$ are reported in ppm relative to $\mathrm{CDCl}_{3}(\delta=7.26)$ for ${ }^{1} \mathrm{H}$ NMR and relative to the central resonance of $\mathrm{CDCl}_{3}(\delta=77.0)$ for ${ }^{13} \mathrm{C} \mathrm{NMR} .{ }^{13} \mathrm{C}$ NMR spectra were acquired on a broad band decoupled mode. Mass spectra were recorded using electrospray $\left(\mathrm{ES}^{+}\right)$ionization techniques. Analytical thin layer chromatography (TLC) was performed using pre-coated aluminium-backed plates and visualized by ultraviolet irradiation or $\mathrm{KMnO}_{4}$ dip unless otherwise stated. Optical rotations were measured on a polarimeter. The enantiomeric excess (ee) of the products was determined by chiral stationary phase HPLC (Daicel Chiralpak AD and AS or Daicel Chiralcel OD columns) or a GC Chrompak CP-Chirasil Dex CB-column.

Materials. Analytical grade solvents were used as received. For flash chromatography (FC) silica gel from Iatron Laboratories Inc. (Iatrobeads 6RS-8060) or silica gel 60, 230400 mesh was used. Catalysts used in the screening are commercially available and were used as received unless otherwise stated. Catalyst VI and VII were prepared using a platinum catalyzed hydrosilation. ${ }^{1}$ Alkylidenes $\mathbf{4 a - j}$ were prepared from the corresponding aldehydes and $\alpha$-cyanoacetates by a literature method. ${ }^{2}$ Acrolein 5 was used as received. 


\section{2-Cyano-2-propenyl-pentanedioic acid dimethyl ester 8}

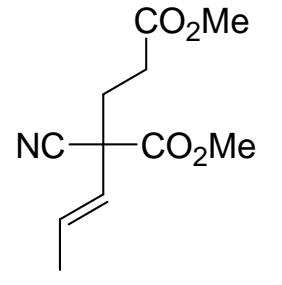

The crude Michael product 6a $(0.40 \mathrm{mmol}$ scale reaction $)$ was dissolved in $5 \% \mathrm{H}_{2} \mathrm{O}$ in $t-\mathrm{BuOH}(3.84 \mathrm{~mL}) . \mathrm{NaClO}_{2}(144 \mathrm{mg}, 1.59$ mmol), $\mathrm{KH}_{2} \mathrm{PO}_{4}(109 \mathrm{mg}, 0.80 \mathrm{mmol})$ and 2-methyl-2-butene (0.34 $\mathrm{mL}, 3.20 \mathrm{mmol})$ were added and the bright yellow solution stirred at room temperature for $90 \mathrm{~min}$. (the reaction was followed by TLC (30\% EtOAc in hexane)). At this time the yellow color had faded and $\mathrm{H}_{2} \mathrm{O}(10 \mathrm{~mL})$ was added. The mixture was extracted with EtOAc $(5 \times 10 \mathrm{~mL})$ and the organic layers were dried $\left(\mathrm{MgSO}_{4}\right)$ and concentrated under reduced pressure to give the corresponding acid. The crude acid under argon was dissolved in $30 \% \mathrm{MeOH}$ in toluene $(11 \mathrm{~mL}) .2 \mathrm{M} \mathrm{TMSCHN}_{2}$ in hexane $(0.59 \mathrm{~mL}, 1.18 \mathrm{mmol})$ was added dropwise with evolution of nitrogen. After complete addition the yellow color of the excess $\mathrm{TMSCHN}_{2}$ remained and the solution was stirred at room temperature for $20 \mathrm{~min}$. The consumption of the acid was monitored by TLC (25\% EtOAc in hexane) developing the plate with bromocresol green with which the acid appears yellow. The reaction was quenched with a few drops of concentrated acetic acid (more nitrogen evolved) and evaporated to give a brown oil. The crude diester was purified by $\mathrm{FC}$ on Iatorobeads (eluent: Gradual polarity changes from DCM to 5\% $\mathrm{Et}_{2} \mathrm{O}$ in $\mathrm{DCM}$ ) to give 2-cyano-2-propenyl-pentanedioic acid dimethyl ester 8 as a clear

oil (43 mg, 48\% overall yield). ${ }^{1} \mathrm{H}$ NMR $\left(\mathrm{CDCl}_{3}\right) \delta 6.07(\mathrm{dq}, J 6.6,15.3 \mathrm{~Hz}, 1 \mathrm{H}), 5.41$ $(\mathrm{dd}, J 1.7,15.4 \mathrm{~Hz}, 1 \mathrm{H}), 3.80(\mathrm{~s}, 3 \mathrm{H}), 3.66(\mathrm{~s}, 3 \mathrm{H}), 2.29-2.54(\mathrm{~m}, 3 \mathrm{H}), 2.14-2.24(\mathrm{~m}, 1 \mathrm{H})$, $1.75(\mathrm{dd}, J 1.7,6.6 \mathrm{~Hz}, 3 \mathrm{H}) .{ }^{13} \mathrm{C} \mathrm{NMR}\left(\mathrm{CDCl}_{3}\right) \delta 172.0,167.9,131.2,124.3,117.0,53.8$, 51.9, 50.9, 32.2, 29.7, 17.6. HRMS: $\mathrm{C}_{11} \mathrm{H}_{15} \mathrm{NO}_{4}[\mathrm{M}+\mathrm{Na}]^{+}$calcd.: 248.0893, found: 248.0888. $[\alpha]_{\mathrm{D}}{ }^{20}+17.5\left(\mathrm{c}=1.0, \mathrm{CHCl}_{3}, 52 \%\right.$ ee $)$. GC Chrompak CP-Chirasil Dex CB- 
column. Temperature program: from $70{ }^{\circ} \mathrm{C}$ to $100{ }^{\circ} \mathrm{C}$ at a rate of $5{ }^{\circ} \mathrm{C} / \mathrm{min}$ maintained temperature for $60 \mathrm{~min}$. then to $180{ }^{\circ} \mathrm{C}$ at a rate of $10{ }^{\circ} \mathrm{C} / \mathrm{min} . \tau_{\text {major }}=70.8 \mathrm{~min}, \tau_{\text {minor }}=$ $70.6 \min (52 \%$ ee $)$.

\section{1-Benzyl-2-oxo-3-propenyl-piperidine-3-carbonitrile 9}

The desired lactam $\mathbf{9}$ and amine $\mathbf{1 4}$ were formed under the described reductive amination conditions. ${ }^{3}$ A solvent screen was carried out in order to limit the formation of $14{ }^{a}$

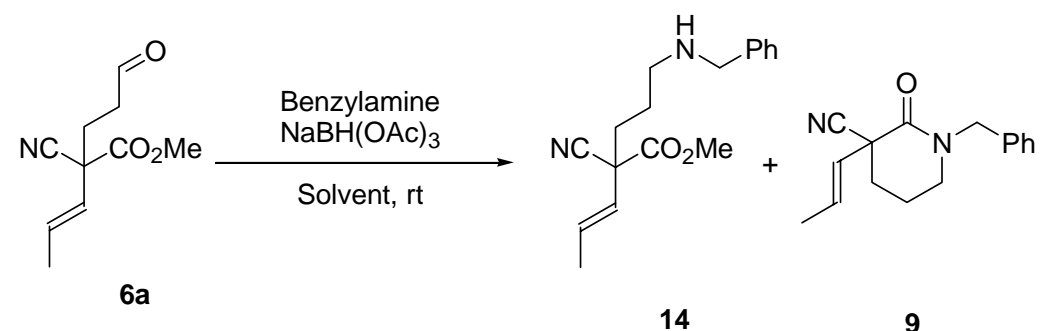

\begin{tabular}{|c|c|c|c|}
\hline Solvent & $\begin{array}{c}\text { Eq. of } \\
\text { NaBH(OAc) }\end{array}$ & $\begin{array}{c}\text { Time } \\
(\mathrm{h})\end{array}$ & $\begin{array}{c}\text { Amine 14:Lactam 9 } \\
\text { ratio }\end{array}$ \\
\hline DCE & 1.4 & $2-3$ & $67: 33$ \\
\hline DCE & 2.5 & 20 & $73: 27$ \\
\hline THF & 1.4 & 19 & $20: 80$ \\
\hline $\mathrm{MeCN}$ & 1.4 & 20 & $50: 50$ \\
\hline Dioxane & 1.4 & 18 & $17: 83(7: 93)^{b}$ \\
\hline TBME & 1.4 & 18 & $50: 50$ \\
\hline $\mathrm{Et}_{2} \mathrm{O}$ & 1.4 & 18 & $36: 64$ \\
\hline
\end{tabular}

$a$ Catalytic reaction performed with $\mathrm{Et}_{3} \mathrm{~N}$ as the catalyst. $b$ The number in the parenthesis refers to the non-racemic case employing the standard conditions for the catalytic step with VII as the catalyst and 1.5 eq. acrolein 5 (TBME: Tertiary butyl methyl ether). 
The crude Michael product 6a $(0.25 \mathrm{mmol}$ scale reaction $)$ was<smiles>C/C=C/C1(C#N)CCCN(Cc2ccccc2)C1=O</smiles>
dissolved in dry 1,4-dioxane $(1 \mathrm{~mL})$. Benzylamine $(27 \mu \mathrm{L}, 0.25$ mmol) and $\mathrm{NaBH}(\mathrm{OAc})_{3}(74 \mathrm{mg}, 0.35 \mathrm{mmol})$ were added and the cloudy mixture stirred under argon at room temperature for $21 \mathrm{~h}$. Aqueous saturated $\mathrm{NaHCO}_{3}$ was added and the mixture extracted with EtOAc $(4 \times 2$ $\mathrm{mL}$ ), after drying $\left(\mathrm{Na}_{2} \mathrm{SO}_{4}\right)$ the organic layers were condensed to give a clear oil. The crude lactam was purified by $\mathrm{FC}$ on $\mathrm{SiO}_{2}$ (eluent: Gradual polarity change from hexane to $30 \%$ EtOAc in hexane) to give 1-benzyl-2-oxo-3-propenyl-piperidine-3-carbonitrile 9 as a clear oil $\left(25 \mathrm{mg}, 39 \%\right.$, three steps). ${ }^{1} \mathrm{H}$ NMR $\left(\mathrm{CDCl}_{3}\right) \delta 7.28-7.39(\mathrm{~m}, 5 \mathrm{H}), 6.07$ (dq, $J$ 6.6, $15.3 \mathrm{~Hz}, 1 \mathrm{H}), 5.58$ (dd, J 1.6, $15.3 \mathrm{~Hz}, 1 \mathrm{H}), 4.63(\mathrm{~d}, J 14.6 \mathrm{~Hz}, 1 \mathrm{H}), 4.57$ (d, $J 14.5$ Hz, 1H), 3.20-3.34 (m, 2H), 2.26-2.34 (m, 1H), 1.87-2.12 (m, 3H), 1.80 (dd, J 1.6, 6.6 $\mathrm{Hz}, 3 \mathrm{H}) .{ }^{13} \mathrm{C} \mathrm{NMR}\left(\mathrm{CDCl}_{3}\right) \delta 164.1,136.1,130.4,128.7,128.1,127.7,126.3,119.6$, 51.1, 47.02, 46.99, 33.2, 19.1, 17.8. HRMS: $\mathrm{C}_{16} \mathrm{H}_{18} \mathrm{~N}_{2} \mathrm{O}[\mathrm{M}+\mathrm{Na}]^{+}$calcd.: 277.1311, found: 277.1313. $[\alpha]_{\mathrm{D}}^{20}+3.1\left(\mathrm{c}=1.0, \mathrm{CHCl}_{3}, 55 \%\right.$ ee). HPLC: Daicel Chiralpak AS column [hexane/iPrOH (90:10)]; flow rate $1.0 \mathrm{~mL} / \mathrm{min}\left(\tau_{1}=16.5 \mathrm{~min} . ; \tau_{2}=27.1 \mathrm{~min}\right.$.) $(55 \%$ ee $)$.

\section{2-Hydroxymethyl-2-(3-hydroxy-propyl)-pent-3-enenitrile 10}

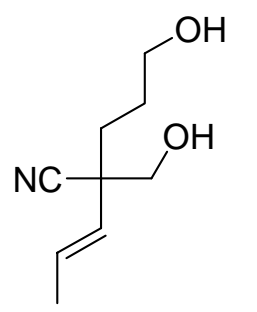

Michael product 6a $(0.20 \mathrm{mmol}$ scale reaction) was dissolved in $1 / 1 \mathrm{CH}_{2} \mathrm{Cl}_{2} / \mathrm{MeOH}(2 \mathrm{~mL})$ and cooled to $-20{ }^{\circ} \mathrm{C} . \mathrm{NaBH}_{4}(0.40$ mmol) was added and the reaction stirred at this temperature for $18 \mathrm{~h}$. The reaction mixed was diluted with $\mathrm{H}_{2} \mathrm{O}(2 \mathrm{~mL})$ and the crude product extracted with $\mathrm{CH}_{2} \mathrm{Cl}_{2}(3 \times 10 \mathrm{~mL})$. The organic 
layers were dried $\left(\mathrm{MgSO}_{4}\right)$, concentrated under reduced pressure and the crude diol purified by $\mathrm{FC}$ on $\mathrm{SiO}_{2}$ eluting with $\mathrm{Et}_{2} \mathrm{O} / \mathrm{MeOH} 10 / 1$ to give $\mathbf{1 0}$ as a clear oil $(17 \mathrm{mg}$, $49 \%$ overall yield). ${ }^{1} \mathrm{H}$ NMR $\left(\mathrm{CDCl}_{3}\right) \delta 6.01(\mathrm{dq}, J$ 6.4, $15.4 \mathrm{~Hz}, 1 \mathrm{H}), 5.18(\mathrm{dd}, J$ 1.6, $15.4 \mathrm{~Hz}, 1 \mathrm{H}), 3.69$ (t, J 5.9 Hz, 2H), 3.64 (d, J 3.4 Hz, 2H), 1.85-1.97 (m, 1H), 1.78 (dd, $J$ 1.6, $6.5 \mathrm{~Hz}, 3 \mathrm{H}), 1.70(\mathrm{~m}, 2 \mathrm{H}), 1.55-1.64(\mathrm{~m}, 1 \mathrm{H}) .{ }^{13} \mathrm{C} \mathrm{NMR}\left(\mathrm{CDCl}_{3}\right) \delta 130.6,126.6$, 121.0, 67.5, 62.2, 47.6, 31.0, 27.8, 17.8. HRMS: $\mathrm{C}_{9} \mathrm{H}_{15} \mathrm{NO}_{2}[\mathrm{M}+\mathrm{Na}]^{+}$calcd.: 192.0995, found: $192.0986 .[\alpha]_{\mathrm{D}}{ }^{20}+0.6\left(\mathrm{c}=1.0 \mathrm{CH}_{2} \mathrm{Cl}_{2}, 56 \%\right.$ ee $)$.

\section{2-Cyano-2-(3-hydroxy-propyl)-pent-3-enoic acid methyl ester 11}

Michael product 6a $(0.30 \mathrm{mmol}$ scale reaction) was dissolved in

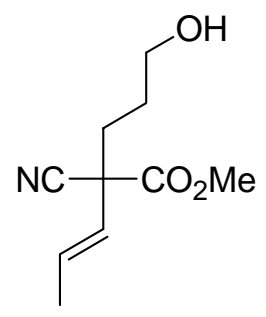

4/1 THF/AcOH $(2.5 \mathrm{~mL})$ and cooled to $-20{ }^{\circ} \mathrm{C} . \mathrm{NaCNBH}_{3}(0.45$ mmol) was added and the reaction stirred at this temperature for $18 \mathrm{~h}$. The reaction mixed was diluted with $\mathrm{H}_{2} \mathrm{O}(4 \mathrm{~mL})$ and the crude product extracted with $\mathrm{NaHCO}_{3} / \mathrm{Et}_{2} \mathrm{O}(4 \times 15 \mathrm{~mL})$. The organic layers were dried $\left(\mathrm{MgSO}_{4}\right)$ and concentrated under reduced pressure to give the corresponding alcohol. The crude alcohol was purified by $\mathrm{FC}$ on $\mathrm{SiO}_{2}$ eluting with $\mathrm{CH}_{2} \mathrm{Cl}_{2}$ to remove traces of $\mathbf{6 a}$ followed by $\mathrm{CH}_{2} \mathrm{Cl}_{2} / \mathrm{Et}_{2} \mathrm{O} 10 / 1$ to give $\mathbf{1 1}$ as a clear oil (51 mg, 87\% overall yield). ${ }^{1} \mathrm{H}$ NMR $\left(\mathrm{CDCl}_{3}\right) \delta 6.08(\mathrm{dq}, J 6.4,15.2 \mathrm{~Hz}, 1 \mathrm{H}), 5.44$ (dd, $J$ 1.6, $15.4 \mathrm{~Hz}, 1 \mathrm{H}), 3.82$ (s, 3H), 3.69 (t, J 6.2 Hz, 2H), 2.15 (dt, J 4.9, $12.2 \mathrm{~Hz}, 1 \mathrm{H}), 1.93$ (dt, $J$ 4.5, $13.0 \mathrm{~Hz}, 1 \mathrm{H}), 1.77$ (dd, $J$ 1.6, $6.7 \mathrm{~Hz}, 3 \mathrm{H}), 1.70-1.82(\mathrm{~m}, 1 \mathrm{H}), 1.64(\mathrm{~m}, 1 \mathrm{H})$, $1.53($ broad s, $1 \mathrm{H}) .{ }^{13} \mathrm{C} \mathrm{NMR}\left(\mathrm{CDCl}_{3}\right) \delta 168.5,130.4,125.0,117.6,61.7,53.7,51.5,34.1$, 28.2, 17.6. HRMS: $\mathrm{C}_{10} \mathrm{H}_{15} \mathrm{NO}_{3}[\mathrm{M}+\mathrm{Na}]^{+}$calcd.: 220.0944 , found: $220.0937 .[\alpha]_{\mathrm{D}}{ }^{20}+10.7$ $\left(\mathrm{c}=1.0, \mathrm{CHCl}_{3}, 30 \%\right.$ ee). GC Chrompak CP-Chirasil Dex CB-column. Temperature 
program: from $70{ }^{\circ} \mathrm{C}$ to $140{ }^{\circ} \mathrm{C}$ at a rate of $10^{\circ} \mathrm{C} / \mathrm{min}$ maintained temperature for $10 \mathrm{~min}$. then to $180^{\circ} \mathrm{C}$ at a rate of $10^{\circ} \mathrm{C} / \mathrm{min} . \tau_{\text {major }}=15.9 \mathrm{~min}, \tau_{\text {minor }}=16.5 \mathrm{~min}(56 \%$ ee $)$.

\section{2-Oxo-3-propenyl-tetrahydro-pyran-3-carbonitrile 12}

To the purified alcohol $11(31 \mathrm{mg}, 0.16 \mathrm{mmol})$ in DCE $(1.25 \mathrm{~mL}), \mathrm{SiO}_{2}$<smiles>C/C=C/C1(C#N)CCCOC1=O</smiles>
$(620 \mathrm{mg})$ was added. The mixture was stirred in a closed vial at $70{ }^{\circ} \mathrm{C}$ for 5 days before being cooled to room temperature and passed through a short plug of $\mathrm{SiO}_{2}$ using $5 \% \mathrm{Et}_{2} \mathrm{O}$ in $\mathrm{DCM}$ as the eluent to remove unreacted 11. After evaporation of the solvent the pure lactone $\mathbf{1 2}$ was obtained as a clear oil (6.1 mg, 23\%, one step). ${ }^{1} \mathrm{H}$ NMR $\left(\mathrm{CDCl}_{3}\right) \delta 6.08(\mathrm{dq}, J 6.6,15.3 \mathrm{~Hz}, 1 \mathrm{H}), 5.55(\mathrm{dd}, J$ 1.7, $15.3 \mathrm{~Hz}, 1 \mathrm{H}), 4.41-4.58(\mathrm{~m}, 2 \mathrm{H}), 2.37-2.47(\mathrm{~m}, 1 \mathrm{H}), 2.17-2.27(\mathrm{~m}, 1 \mathrm{H}), 2.01-2.13$ (m, 2H), $1.81(\mathrm{dd}, J 1.7,6.6 \mathrm{~Hz}, 3 \mathrm{H}) .{ }^{13} \mathrm{C} \mathrm{NMR}\left(\mathrm{CDCl}_{3}\right) \delta 164.8,132.0,124.6,117.9$, 70.3, 46.0, 32.1, 19.3, 17.8. MS: $\mathrm{C}_{9} \mathrm{H}_{11} \mathrm{NO}_{2}[\mathrm{M}+\mathrm{Na}]^{+}$calcd.: 188.07, found: 188.1 (The

compound is too unstable for determination of the exact mass). $[\alpha]_{\mathrm{D}}{ }^{20}+3.6(\mathrm{c}=0.47$, $\mathrm{CHCl}_{3}, 55 \%$ ee). GC Chrompak CP-Chirasil Dex CB-column. Temperature program: from $70{ }^{\circ} \mathrm{C}$ to $100{ }^{\circ} \mathrm{C}$ at a rate of $5{ }^{\circ} \mathrm{C} / \mathrm{min}$ maintained temperature for $30 \mathrm{~min}$. then to 180 ${ }^{\circ} \mathrm{C}$ at a rate of $10{ }^{\circ} \mathrm{C} / \mathrm{min} . \tau_{\text {major }}=42.8 \mathrm{~min}, \tau_{\text {minor }}=43.0 \mathrm{~min}(55 \%$ ee $)$. 


\section{2-(3-Hydroxy-propyl)-pent-3-enenitrile 13}

The crude Michael product 6a $(0.125 \mathrm{mmol}$ scale reaction $)$ in dry

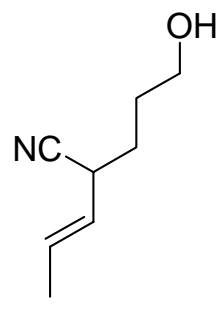

THF (1 mL) was added dropwise to a suspension of $\mathrm{LiAlH}_{4}(14$ $\mathrm{mg}, 0.369 \mathrm{mmol})$ in dry THF $(1 \mathrm{~mL})$ at $-78{ }^{\circ} \mathrm{C}$. The mixture was stirred under argon at $-78{ }^{\circ} \mathrm{C}$ for $15 \mathrm{~min}$. then quenched with MeOH $(1 \mathrm{~mL})$ then $\mathrm{H}_{2} \mathrm{O}(3 \mathrm{~mL})$. The mixture was extracted with

EtOAc $(4 \times 5 \mathrm{~mL})$ and the organic layers were washed with brine $(15 \mathrm{~mL})$ and dried $\left(\mathrm{MgSO}_{4}\right)$ before being concentrated to a brown oil. The crude product was purified by $\mathrm{FC}$ on $\mathrm{SiO}_{2}$ (eluent: $15 \% \mathrm{Et}_{2} \mathrm{O}$ in $\mathrm{CH}_{2} \mathrm{Cl}_{2}$ ) to give racemic 2-(3-hydroxy-propyl)-pent-3enenitrile 13 as a clear oil (9 mg, 52\%, two steps). ${ }^{1} \mathrm{H}$ NMR $\left(\mathrm{CDCl}_{3}\right) \delta 5.83$ (dq, $J 6.5$, $15.2 \mathrm{~Hz}, 1 \mathrm{H}), 5.34$ (ddd, J 1.7, 6.4, $15.3 \mathrm{~Hz}, 1 \mathrm{H}), 3.65-3.73(\mathrm{~m}, 2 \mathrm{H}), 3.26$ (q , J $6.1 \mathrm{~Hz}$, $1 \mathrm{H}), 1.66-1.82(\mathrm{~m}, 7 \mathrm{H}), 1.60($ broad s, $1 \mathrm{H}) .{ }^{13} \mathrm{C} \mathrm{NMR}\left(\mathrm{CDCl}_{3}\right) \delta 129.8,124.6,120.5$, 61.9, 33.8, 29.7, 29.6, 17.6. HRMS: $\mathrm{C}_{8} \mathrm{H}_{13} \mathrm{NO}[\mathrm{M}+\mathrm{Na}]^{+}$calcd.:162.0889, found: 162.0889. GC Chrompak CP-Chirasil Dex CB-column. Temperature program: from 70 ${ }^{\circ} \mathrm{C}$ to $100{ }^{\circ} \mathrm{C}$ at a rate of $5^{\circ} \mathrm{C} / \mathrm{min}$ maintained temperature for $30 \mathrm{~min}$. then to $180{ }^{\circ} \mathrm{C}$ at a rate of $10{ }^{\circ} \mathrm{C} / \mathrm{min} . \tau=8.6 \mathrm{~min}, \tau=8.9 \mathrm{~min}(0 \%$ ee $)$. 


\section{2-Cyano-2-(3-phenylamino-propyl)-dec-3-enoic acid methyl ester 7b}

The title compound was obtained according to the general

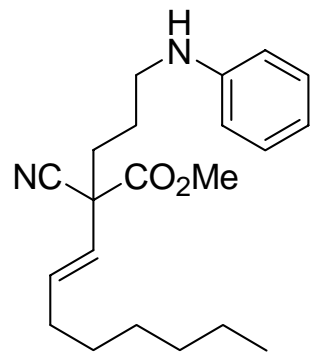

procedure (reaction time $19 \mathrm{~h}$ ) as a colourless oil, yield 52\% (two steps). ${ }^{1} \mathrm{H}$ NMR $\left(\mathrm{CDCl}_{3}\right) \oint 7.18(\mathrm{t}, J 7.5 \mathrm{~Hz}, 2 \mathrm{H}), 6.72(\mathrm{dt}, J$ 1.0, $7.3 \mathrm{~Hz}, 1 \mathrm{H}), 6.61$ (dd, J 1.0, 7.6 Hz, 2H), 6.06 (td, J 6.8, $15.3 \mathrm{~Hz}$, 1H), $5.41(\mathrm{dd}, J 1.0,15.4 \mathrm{~Hz}, 1 \mathrm{H}), 3.79$ (s, 3H), $3.18(\mathrm{t}, J 6.8 \mathrm{~Hz}$, 2H), $2.17(\mathrm{~m}, 1 \mathrm{H}), 2.08$ (dd, J 7.0, $14.4 \mathrm{~Hz}, 2 \mathrm{H}), 1.94(\mathrm{~m}, 1 \mathrm{H})$, $1.83(\mathrm{~m}, 1 \mathrm{H}), 1.70(\mathrm{~m}, 1 \mathrm{H}), 1.38(\mathrm{dd}, J 6.8,13.9 \mathrm{~Hz}, 2 \mathrm{H}), 1.20-1.33(\mathrm{~m}, 6 \mathrm{H}), 0.88(\mathrm{t}, J$ $6.8 \mathrm{~Hz}, 3 \mathrm{H}) .{ }^{13} \mathrm{C} \mathrm{NMR}\left(\mathrm{CDCl}_{3}\right) \delta 168.4,147.9,135.8,129.3,123.7,117.7,117.5,112.7$, 53.7, 51.6, 43.1, 35.2, 32.0, 31.5, 28.7, 28.6, 25.3, 22.5, 14.0. HRMS: $\mathrm{C}_{21} \mathrm{H}_{30} \mathrm{~N}_{2} \mathrm{O}_{2}$ $[\mathrm{M}+\mathrm{Na}]^{+}$calc.: 365.2199 , found: $365.2205 .[\alpha]_{\mathrm{D}}{ }^{20}+15.0\left(\mathrm{c}=1.0, \mathrm{CH}_{2} \mathrm{Cl}_{2}, 56 \%\right.$ ee $)$. The ee was determined by HPLC using a Chiralcel OD column [hexane/iPrOH (90:10)]; flow rate $1.0 \mathrm{~mL} / \mathrm{min} ; \tau_{\text {major }}=19.8 \mathrm{~min}, \tau_{\operatorname{minor}}=15.5 \mathrm{~min}(56 \%$ ee $)$.

\section{2-Cyano-2-(3-phenylamino-propyl)-hepta-3,6-dienoic acid methyl ester 7c}

The title compound was obtained according to the general

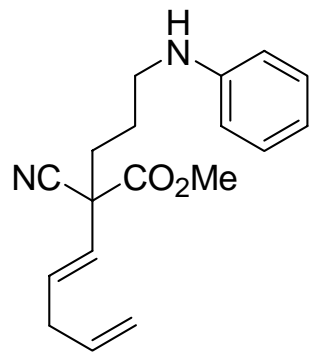

procedure (reaction time $18 \mathrm{~h}$ ) as a colourless oil, yield 54\% (two steps). ${ }^{1} \mathrm{H}$ NMR $\left(\mathrm{CDCl}_{3}\right) \oint 7.18(\mathrm{t}, J 7.5 \mathrm{~Hz}, 2 \mathrm{H}), 6.71(\mathrm{dt}, J$ 1.0, $7.3 \mathrm{~Hz}, 1 \mathrm{H}) 6.59(\mathrm{dd}, J 1.0,7.7 \mathrm{~Hz}, 2 \mathrm{H}), 6.11(\mathrm{td}, J$ 6.5, $15.4 \mathrm{~Hz}$, 1H), $5.79(\mathrm{~m}, 1 \mathrm{H}), 5.46(\mathrm{dd}, J 1.0,15.5 \mathrm{~Hz}, 1 \mathrm{H}), 5.08(\mathrm{~m}, 1 \mathrm{H})$, $5.04(\mathrm{dd}, J 1.4,5.9 \mathrm{~Hz}, 1 \mathrm{H}), 3.80(\mathrm{~s}, 3 \mathrm{H}), 3.18(\mathrm{t}, J 6.8 \mathrm{~Hz}, 2 \mathrm{H})$,

$2.85(\mathrm{dt}, J 1.3,6.5 \mathrm{~Hz}, 2 \mathrm{H}), 2.19(\mathrm{~m}, 1 \mathrm{H}), 1.95(\mathrm{~m}, 1 \mathrm{H}), 1.84(\mathrm{~m}, 1 \mathrm{H}), 1.70(\mathrm{~m}, 1 \mathrm{H}) .{ }^{13} \mathrm{C}$ $\operatorname{NMR}\left(\mathrm{CDCl}_{3}\right) \delta 168.2,147.9,134.8,133.2,129.3,124.9,117.54,117.50,116.6,112.7$ 
53.7, 51.6, 43.1, 35.9, 35.3, 25.3. HRMS: $\mathrm{C}_{18} \mathrm{H}_{22} \mathrm{~N}_{2} \mathrm{O}_{2}[\mathrm{M}+\mathrm{H}]^{+}$calc.: 299.1754, found: 299.1755. $[\alpha]_{\mathrm{D}}^{20}+10.1\left(\mathrm{c}=1.0, \mathrm{CH}_{2} \mathrm{Cl}_{2}, 51 \%\right.$ ee). The ee was determined by HPLC using a Chiralcel OD column [hexane $/ \mathrm{PrOH}(90: 10)]$; flow rate $1.0 \mathrm{~mL} / \mathrm{min} ; \tau_{\text {major }}=19.6$ $\min , \tau_{\operatorname{minor}}=24.8 \min (51 \%$ ee $)$.

\section{7-(tert-Butyl-dimethyl-silanyloxy)-2-cyano-2-(3-phenylamino-propyl)-hept-3-enoic acid methyl ester $7 d$}

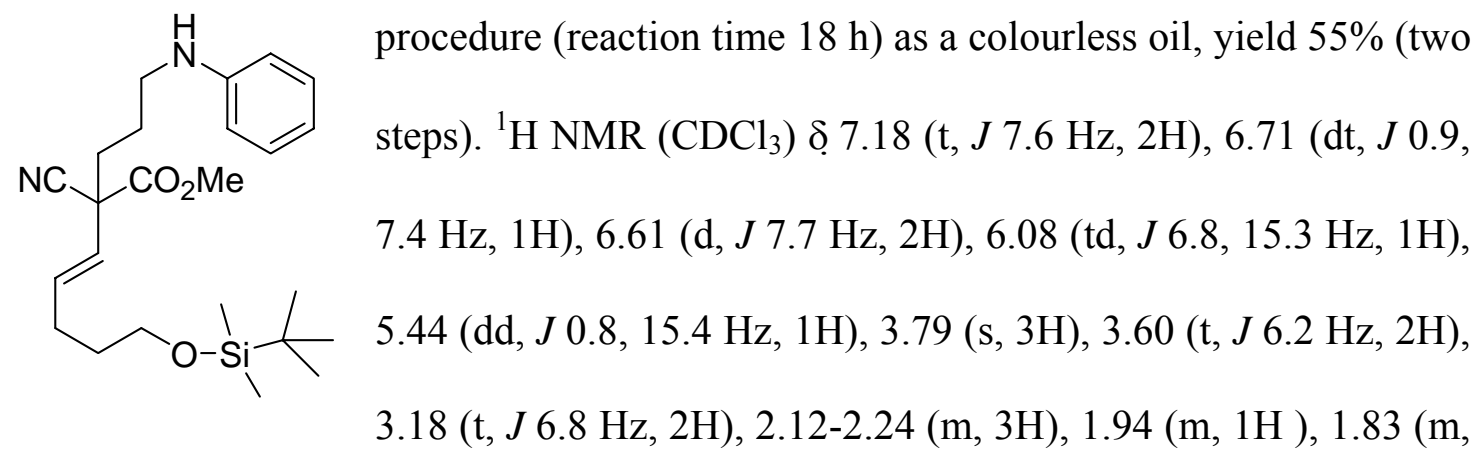

1H), $1.70(\mathrm{~m}, 1 \mathrm{H}), 1.61(\mathrm{td}, J$ 6.5, $13.5 \mathrm{~Hz}, 2 \mathrm{H}), 0.90(\mathrm{~s}, 9 \mathrm{H}), 0.05(\mathrm{~s}, 6 \mathrm{H}) .{ }^{13} \mathrm{C} \mathrm{NMR}$ $\left(\mathrm{CDCl}_{3}\right) \delta 168.3,147.8,135.2,129.2,124.1,117.58,117.55,112.7,62.1,53.7,51.6$, 43.1, 35.3, 31.7, 28.4, 25.9, 25.3, 18.3, -5.4. HRMS: $\mathrm{C}_{24} \mathrm{H}_{38} \mathrm{~N}_{2} \mathrm{O}_{3} \mathrm{Si}[\mathrm{M}+\mathrm{Na}]^{+}$calc.: 453.2544, found: 453.2555. $[\alpha]_{\mathrm{D}}^{20}+11.1\left(\mathrm{c}=1.0, \mathrm{CH}_{2} \mathrm{Cl}_{2}, 53 \%\right.$ ee). The ee was determined by HPLC using a Chiralcel OD column [hexane/iPrOH (90:10)]; flow rate 1.0 $\mathrm{mL} / \mathrm{min} ; \tau_{\text {major }}=16.3 \mathrm{~min}, \tau_{\text {minor }}=13.4 \min (53 \%$ ee $)$. 


\section{2-Cyano-5-methyl-2-(3-phenylamino-propyl)-hex-3-enoic acid methyl ester 7e}

The title compound was obtained according to the general

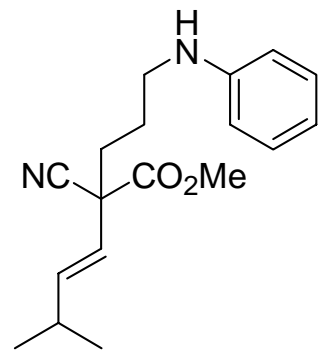

procedure (reaction time $19 \mathrm{~h}$ ) as a colourless oil, yield 63\% (two steps). ${ }^{1} \mathrm{H} \mathrm{NMR}\left(\mathrm{CDCl}_{3}\right) \oint 7.18(\mathrm{dd}, J 7.4,8.2 \mathrm{~Hz}, 2 \mathrm{H}), 6.71(\mathrm{t}, J$ $7.2 \mathrm{~Hz}, 1 \mathrm{H}), 6.60$ (d, J 7.7 Hz, 2H), 6.05 (dd, J 6.7, $15.5 \mathrm{~Hz}, 1 \mathrm{H})$, $5.37(\mathrm{dd}, J 1.2,15.5 \mathrm{~Hz}, 1 \mathrm{H}), 3.80(\mathrm{~s}, 3 \mathrm{H}), 3.18(\mathrm{t}, J 6.8 \mathrm{~Hz}, 2 \mathrm{H})$, $2.37(\mathrm{~m}, 1 \mathrm{H}), 2.18$ (ddd, J 4.8, 11.7, $13.4 \mathrm{~Hz}, 1 \mathrm{H}), 1.94(\mathrm{~m}, 1 \mathrm{H})$,

$1.83(\mathrm{~m}, 1 \mathrm{H}), 1.69(\mathrm{~m}, 1 \mathrm{H}), 1.26(\operatorname{broad} \mathrm{s}, 1 \mathrm{H}), 1.03(\mathrm{~s}, 3 \mathrm{H}), 1.01(\mathrm{~s}, 3 \mathrm{H}) .{ }^{13} \mathrm{C} \mathrm{NMR}$ $\left(\mathrm{CDCl}_{3}\right) \delta 168.5,147.8,142.2,129.2,121.3,117.7,117.6,112.8,53.7,51.6,43.1,35.4$ 30.8, 25.2, 21.9, 21.8. HRMS: $\mathrm{C}_{18} \mathrm{H}_{24} \mathrm{~N}_{2} \mathrm{O}_{2}[\mathrm{M}+\mathrm{Na}]^{+}$calc.: 323.1730, found: 323.1718 . $[\alpha]_{\mathrm{D}}^{20}+16.4\left(\mathrm{c}=1.0, \mathrm{CH}_{2} \mathrm{Cl}_{2}, 50 \%\right.$ ee $)$. The ee was determined by HPLC using a Chiralcel OD column [hexane/iPrOH (99:1)]; flow rate $1.0 \mathrm{~mL} / \mathrm{min} ; \tau_{\text {major }}=81.6 \mathrm{~min}$, $\tau_{\text {minor }}=66.7 \min (50 \%$ ee $)$.

\section{2-Cyano-5,5-dimethyl-2-(3-phenylamino-propyl)-hex-3-enoic acid methyl ester 7f}

The title compound was obtained according to the general

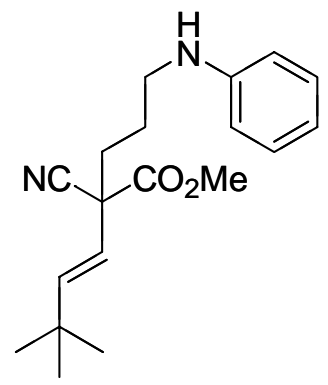
procedure (reaction time $20 \mathrm{~h}$ ) as a colourless oil, yield 59\% (two steps). ${ }^{1} \mathrm{H} \mathrm{NMR}\left(\mathrm{CDCl}_{3}\right) \oint 7.18(\mathrm{t}, J 7.6 \mathrm{~Hz}, 2 \mathrm{H}), 6.73(\mathrm{t}, J 7.4$ Hz, 1H), 6.62 (d, J 7.6 Hz, 2H), 6.08 (d, J $15.7 \mathrm{~Hz}, 1 \mathrm{H}), 5.32$ (d, $J 15.7 \mathrm{~Hz}, 1 \mathrm{H}), 3.80$ (s, 3H), 3.18 (t, J $6.8 \mathrm{~Hz}, 2 \mathrm{H}), 2.18$ (m, 1H), $1.93(\mathrm{~m}, 1 \mathrm{H}), 1.83(\mathrm{~m}, 1 \mathrm{H}), 1.69(\mathrm{~m}, 1 \mathrm{H}), 1.03(\mathrm{~s}, 9 \mathrm{H}){ }^{13} \mathrm{C} \mathrm{NMR}$

$\left(\mathrm{CDCl}_{3}\right) \delta 168.5,147.8,146.0,129.2,119.4,117.7,117.5,112.7,53.7,51.6,43.1,35.5$ 33.4, 29.1, 25.2. HRMS: $\mathrm{C}_{19} \mathrm{H}_{26} \mathrm{~N}_{2} \mathrm{O}_{2}[\mathrm{M}+\mathrm{Na}]^{+}$calc.: 337.1886 , found: $337.1896 .[\alpha]_{\mathrm{D}}{ }^{20}$ 
$+14.6\left(\mathrm{c}=1.0, \mathrm{CH}_{2} \mathrm{Cl}_{2}, 40 \%\right.$ ee $)$. The ee was determined by HPLC using a Chiralpak AD column [hexane $/ \mathrm{PrOH}(99: 1)]$; flow rate $1.0 \mathrm{~mL} / \mathrm{min} ; \tau_{\text {major }}=11.1 \mathrm{~min}, \tau_{\operatorname{minor}}=12.0 \mathrm{~min}$ ( $40 \%$ ee $)$.

\section{2-Cyano-5-phenyl-2-(3-phenylamino-propyl)-pent-3-enoic acid methyl ester 7g}

The title compound was obtained according to the general

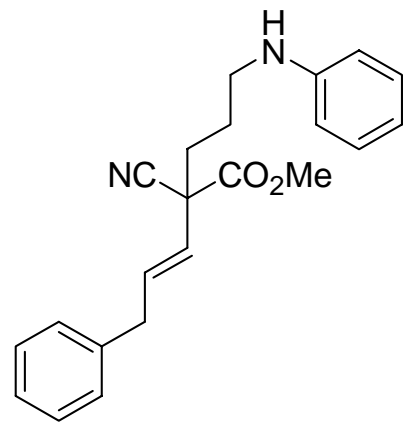

procedure (reaction time $20 \mathrm{~h}$ ) as a colourless oil, yield 39\% (two steps). ${ }^{1} \mathrm{H}$ NMR $\left(\mathrm{CDCl}_{3}\right) \delta 7.32(\mathrm{t}, J 7.2 \mathrm{~Hz}, 2 \mathrm{H}), 7.11-$ $7.28(\mathrm{~m}, 5 \mathrm{H}), 6.73(\mathrm{t}, J 7.3 \mathrm{~Hz}, 1 \mathrm{H}), 6.61(\mathrm{~d}, J 7.7 \mathrm{~Hz}, 2 \mathrm{H})$, $6.26(\mathrm{dt}, J 6.8,15.4 \mathrm{~Hz}, 1 \mathrm{H}), 5.50(\mathrm{~d}, J 15.4 \mathrm{~Hz}, 1 \mathrm{H}), 3.81$ (s, 3H), 3.62 (broad s, 1H), 3.44 (d, J $6.7 \mathrm{~Hz}, 2 \mathrm{H}), 3.18$ (t, $J$ $6.7 \mathrm{~Hz}, 2 \mathrm{H}), 2.20(\mathrm{~m}, 1 \mathrm{H}), 1.97(\mathrm{~m}, 1 \mathrm{H}), 1.84(\mathrm{~m}, 1 \mathrm{H}), 1.71(\mathrm{~m}, 1 \mathrm{H}) .{ }^{13} \mathrm{C} \mathrm{NMR}\left(\mathrm{CDCl}_{3}\right)$ $\delta 168.2,147.9,138.5,134.2,129.3,128.6,128.5,126.5,125.2,117.53,117.46,112.7$, 53.7, 51.5, 43.0, 38.2, 35.3, 25.3. HRMS: $\mathrm{C}_{22} \mathrm{H}_{24} \mathrm{~N}_{2} \mathrm{O}_{2}[\mathrm{M}+\mathrm{Na}]^{+}$calcd.: 371.1730 , found: 371.1722. $[\alpha]_{\mathrm{D}}^{20}+7.0\left(\mathrm{c}=0.98, \mathrm{CHCl}_{3}, 39 \%\right.$ ee $)$. The ee was determined by HPLC using a Chiralcel OD column [hexane/iPrOH (90:10)]; flow rate $1.0 \mathrm{~mL} / \mathrm{min} ; \tau_{\text {major }}=43.7 \mathrm{~min}$, $\tau_{\text {minor }}=52.1 \min (39 \%$ ee $)$. 


\section{2-Cyano-2-(3-phenylamino-propyl)-pent-3-enoic acid isopropyl ester $7 \mathrm{~h}$}

The title compound was obtained according to the general

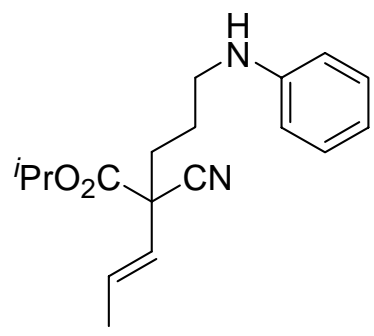

procedure (reaction time $18 \mathrm{~h}$ ) as a colourless oil, yield $52 \%$

(two steps). ${ }^{1} \mathrm{H}$ NMR $\left(\mathrm{CDCl}_{3}\right) \oint 7.18(\mathrm{t}, J 7.5 \mathrm{~Hz}, 2 \mathrm{H}), 6.70$ (dt, $J 1.0,7.4 \mathrm{~Hz}, 1 \mathrm{H}), 6.61(\mathrm{dd}, J 0.9,7.7 \mathrm{~Hz}, 2 \mathrm{H}), 6.08(\mathrm{qd}, J$ 6.6, 15.4 Hz, 1H), 5.44 (dd, J 1.6, $15.4 \mathrm{~Hz}, 1 \mathrm{H}), 5.05$ (septet, $J$

$6.3 \mathrm{~Hz}, 1 \mathrm{H}), 3.66$ (broad s, 1H), 3.18 (t, J $6.8 \mathrm{~Hz}, 2 \mathrm{H}), 2.14$ (dt, J 4.6, $12.6 \mathrm{~Hz}, 1 \mathrm{H}), 1.92$ (m, 1H), $1.83(\mathrm{~m}, 1 \mathrm{H}), 1.77$ (dd, J 1.6, $6.6 \mathrm{~Hz}, 3 \mathrm{H}), 1.70(\mathrm{~m}, 1 \mathrm{H}), 1.29$ (t, J $6.9 \mathrm{~Hz}, 6 \mathrm{H})$. ${ }^{13} \mathrm{C}$ NMR $\left(\mathrm{CDCl}_{3}\right) \delta 167.3,147.8,130.3,129.3,125.1,117.7,117.6,112.8,71.0,51.9$, 43.2, 35.2, 25.2, 21.5, 21.4, 17.6. HRMS: $\mathrm{C}_{18} \mathrm{H}_{24} \mathrm{~N}_{2} \mathrm{O}_{2}[\mathrm{M}+\mathrm{Na}]^{+}$calc. 323.1730:, found: 323.1732. $[\alpha]_{\mathrm{D}}^{20}+19.1\left(\mathrm{c}=1.0, \mathrm{CH}_{2} \mathrm{Cl}_{2}, 55 \%\right.$ ee). The ee was determined by HPLC using a Chiralpak AD column [hexane/ $\mathrm{PrOH}(99: 1)]$; flow rate $1.0 \mathrm{~mL} / \mathrm{min}$; $\tau_{\text {major }}=22.6$ $\min , \tau_{\operatorname{minor}}=28.7 \min (55 \%$ ee $)$.

\section{2-Cyano-2-(3-phenylamino-propyl)-hex-3-enoic acid benzyl ester 7i}

The title compound was obtained according to the general

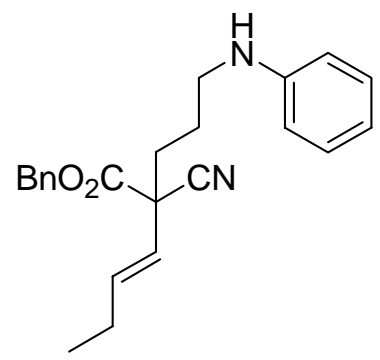
procedure (reaction time $20 \mathrm{~h}$ ) as a colourless oil, yield $39 \%$ (two steps). ${ }^{1} \mathrm{H}$ NMR $\left(\mathrm{CDCl}_{3}\right) \delta$ 7.31-7.42 (m, 5H), $7.18(\mathrm{t}, \mathrm{J}$ $8.0 \mathrm{~Hz}, 2 \mathrm{H}), 6.72(\mathrm{t}, J 7.3 \mathrm{~Hz}, 1 \mathrm{H}), 6.58(\mathrm{~d}, J 7.7 \mathrm{~Hz}, 2 \mathrm{H}), 6.12$ (td, J 6.4, $15.5 \mathrm{~Hz}, 1 \mathrm{H}), 5.40(\mathrm{td}, J 1.6,15.4 \mathrm{~Hz}, 1 \mathrm{H}), 5.22(\mathrm{~s}$, 2H), $3.14(\mathrm{t}, J 6.8 \mathrm{~Hz}, 2 \mathrm{H}), 2.03-2.22(\mathrm{~m}, 3 \mathrm{H}), 1.94(\mathrm{~m}, 1 \mathrm{H})$,

$1.81(\mathrm{~m}, 1 \mathrm{H}), 1.64(\mathrm{~m}, 1 \mathrm{H}), 0.99(\mathrm{t}, J 7.4 \mathrm{~Hz}, 3 \mathrm{H}) .{ }^{13} \mathrm{C} \mathrm{NMR}\left(\mathrm{CDCl}_{3}\right) \delta$ 167.6, 147.6, $137.3,134.6,129.3,128.64,128.63,128.1,122.8,117.75,117.59,112.9,68.2,51.7,43.3$, 
35.2, 25.15, 25.13, 13.0. HRMS: $\mathrm{C}_{23} \mathrm{H}_{26} \mathrm{~N}_{2} \mathrm{O}_{2}[\mathrm{M}+\mathrm{Na}]^{+}$calc. 385.1886:, found: 385.1863 . $[\alpha]_{\mathrm{D}}^{20}+15.9\left(\mathrm{c}=1.0, \mathrm{CH}_{2} \mathrm{Cl}_{2}, 53 \%\right.$ ee $)$. The ee was determined by HPLC using a Chiralcel OD column [hexane $/ \mathrm{iPrOH}(90: 10)]$; flow rate $1.0 \mathrm{~mL} / \mathrm{min} ; \tau_{\text {major }}=56.8 \mathrm{~min}$, $\tau_{\operatorname{minor}}=46.7 \min (53 \%$ ee $)$.

\section{2-Cyano-2-(3-phenylamino-propyl)-hex-3-enoic acid tert-butyl ester 7j}

The title compound was obtained according to the general

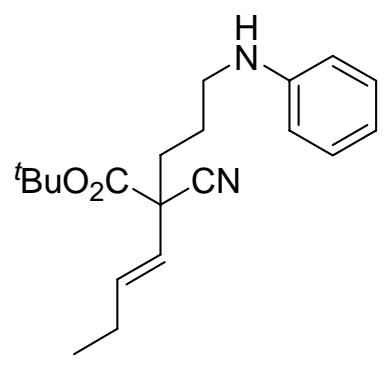

procedure (reaction time $18 \mathrm{~h}$ ) as a colourless oil, yield $45 \%$ (two steps). ${ }^{1} \mathrm{H} \mathrm{NMR}\left(\mathrm{CDCl}_{3}\right) \delta 7.18(\mathrm{dt}, J 1.2,7.4 \mathrm{~Hz}, 2 \mathrm{H})$, $6.71(\mathrm{dt}, J 1.0,7.3 \mathrm{~Hz}, 1 \mathrm{H}), 6.61(\mathrm{dd}, J 1.0,8.6 \mathrm{~Hz}, 2 \mathrm{H}), 6.09$ (td, $J 6.4,15.5 \mathrm{~Hz}, 1 \mathrm{H}), 5.39$ (td, $J 1.6,15.5 \mathrm{~Hz}, 1 \mathrm{H}), 3.18(\mathrm{t}$, J $6.7 \mathrm{~Hz}, 2 \mathrm{H}), 2.12(\mathrm{~m}, 3 \mathrm{H}), 1.87(\mathrm{~m}, 2 \mathrm{H}), 1.72(\mathrm{~m}, 1 \mathrm{H}), 1.48$ (s, 9H), $1.02(\mathrm{t}, J 7.4 \mathrm{~Hz}, 3 \mathrm{H}) .{ }^{13} \mathrm{C} \mathrm{NMR}\left(\mathrm{CDCl}_{3}\right) \delta 166.8,147.8,136.6,129.2,123.5$, 118.1, 117.5, 112.7, 84.1, 52.4, 43.2, 35.2, 27.7, 25.19, 25.17, 13.1. HRMS: $\mathrm{C}_{20} \mathrm{H}_{28} \mathrm{~N}_{2} \mathrm{O}_{2}$ $[\mathrm{M}+\mathrm{Na}]^{+}$calc. 351.2043:, found: $351.2031 .[\alpha]_{\mathrm{D}}^{20}+13.1\left(\mathrm{c}=1.0, \mathrm{CH}_{2} \mathrm{Cl}_{2}, 40 \%\right.$ ee). The ee was determined by HPLC using a Chiralcel AD column [hexane/iPrOH (90:10)]; flow rate $1.0 \mathrm{~mL} / \mathrm{min} ; \tau_{\text {major }}=5.5 \mathrm{~min}, \tau_{\text {minor }}=6.9 \mathrm{~min}(40 \%$ ee $)$. 
${ }^{1} \mathrm{H}$ and ${ }^{13} \mathrm{C}$ NMR Spectra of Compounds 6a, 7a-j, 8, 9, 10, 11, 12 and 13
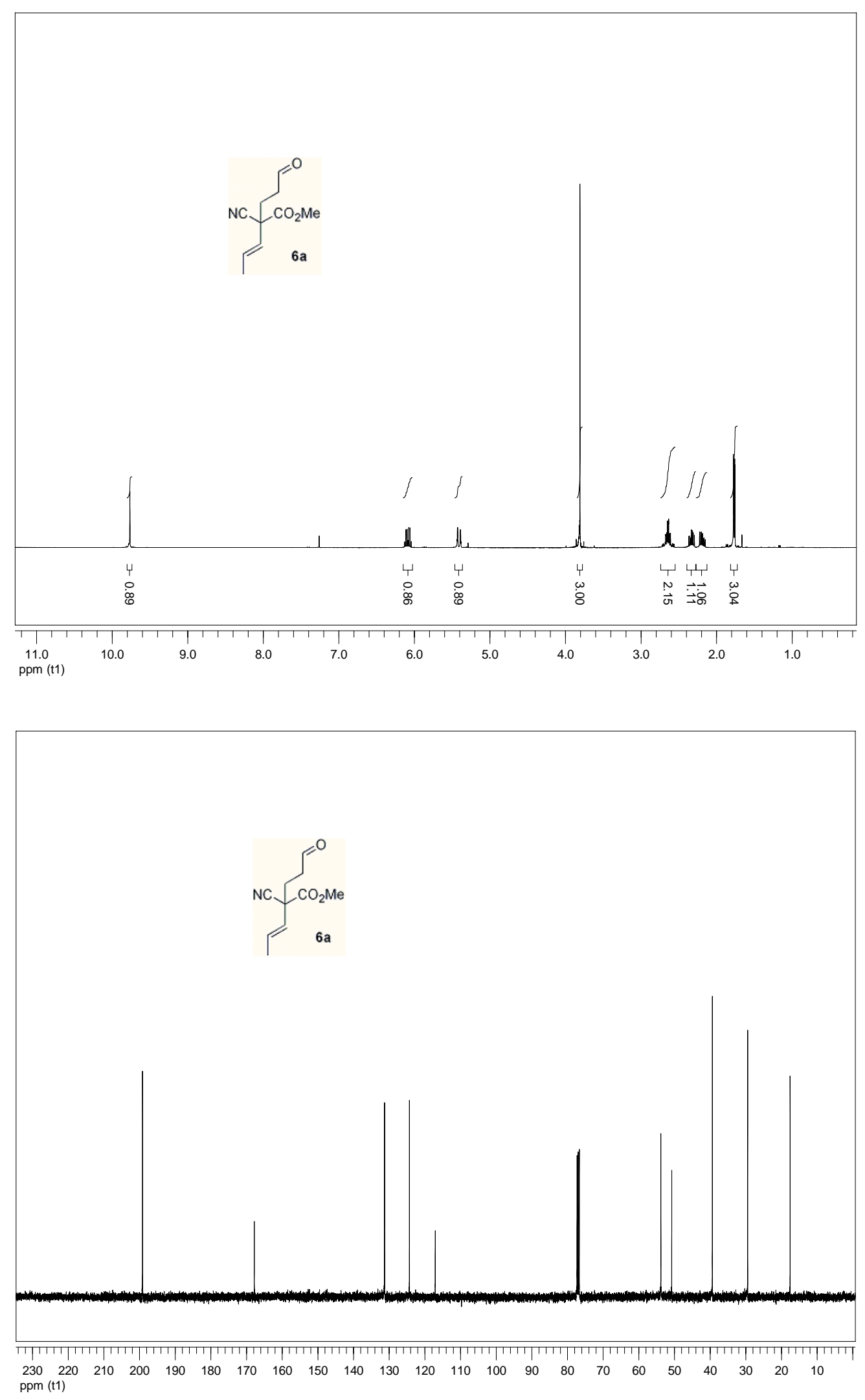

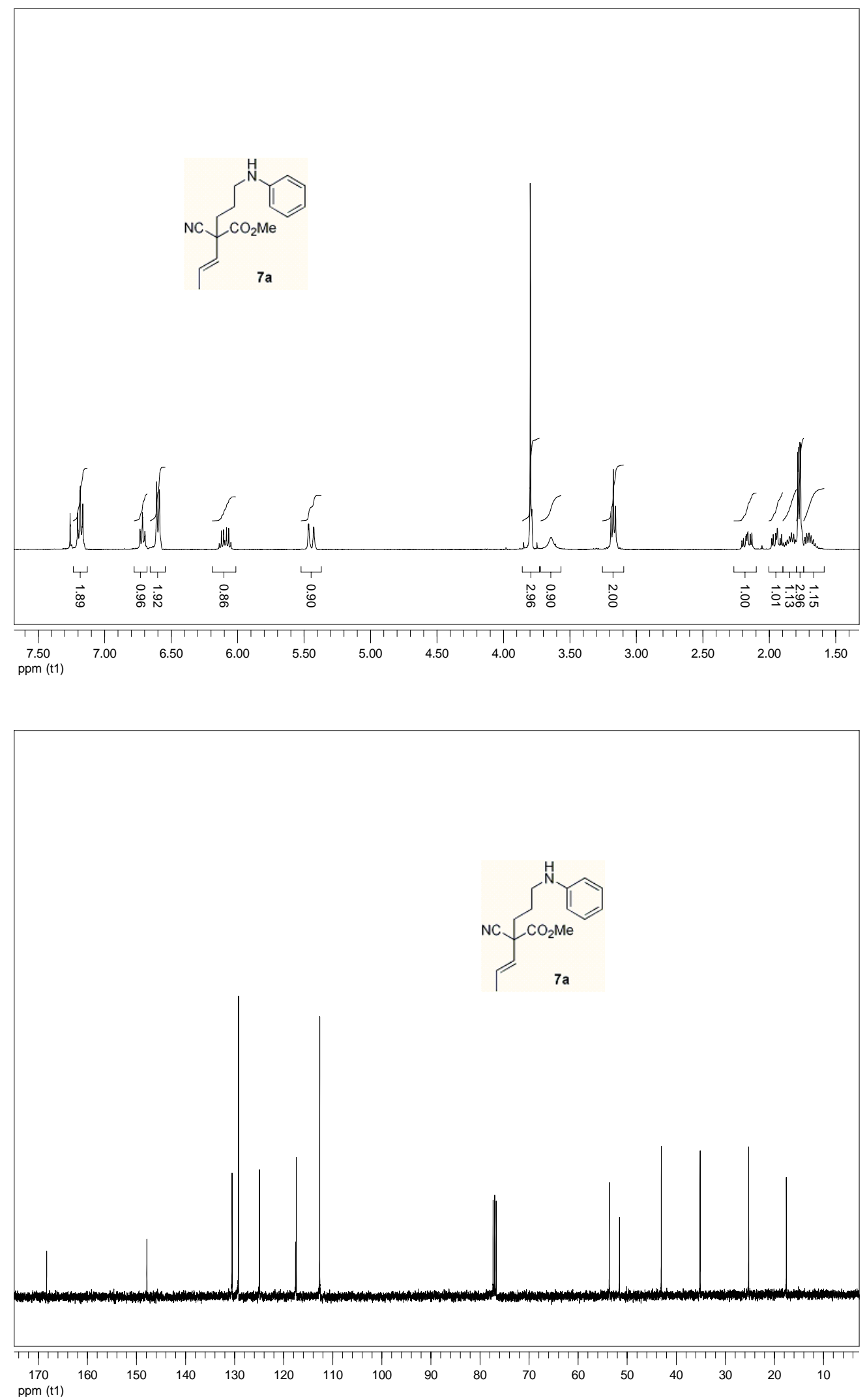

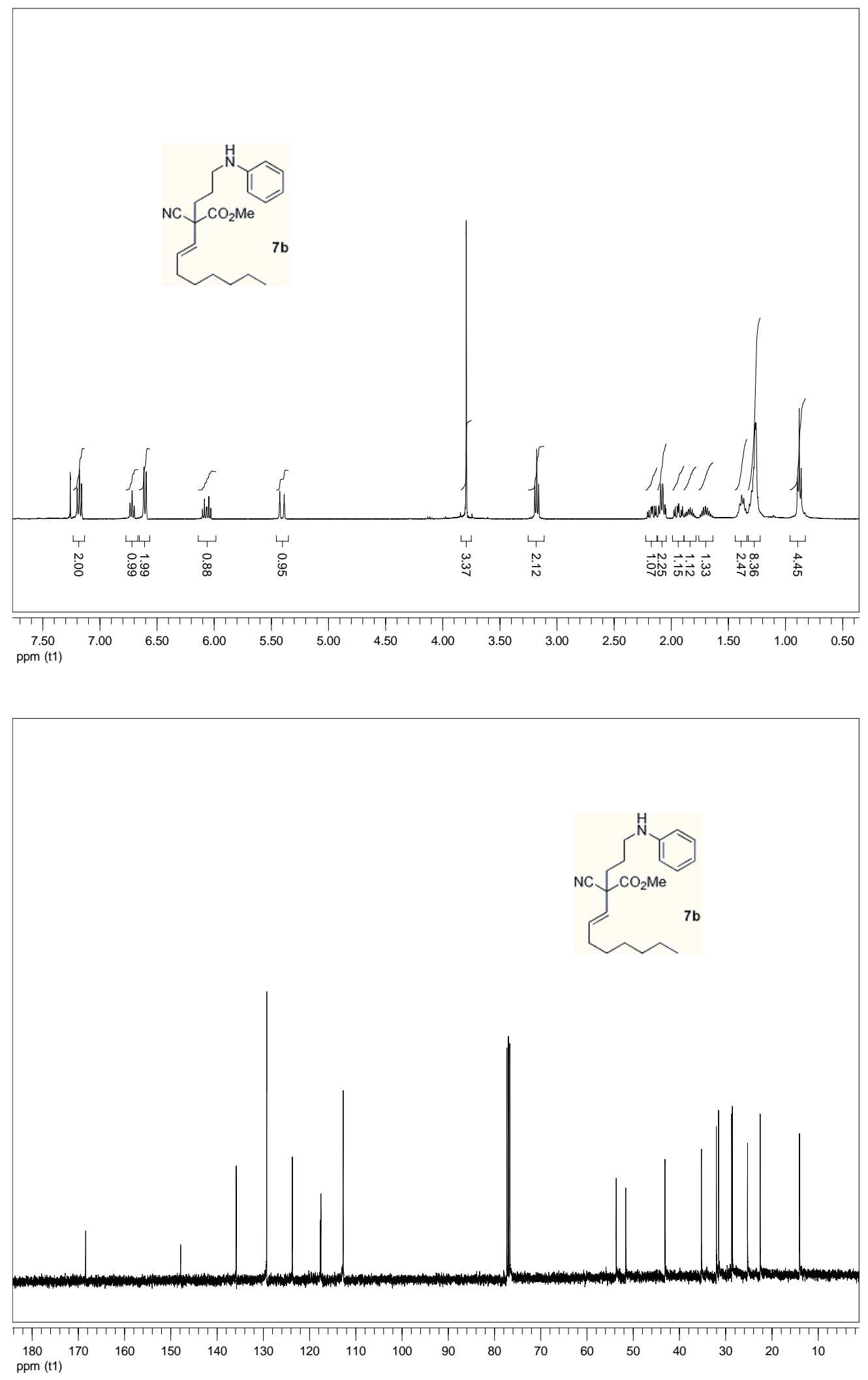

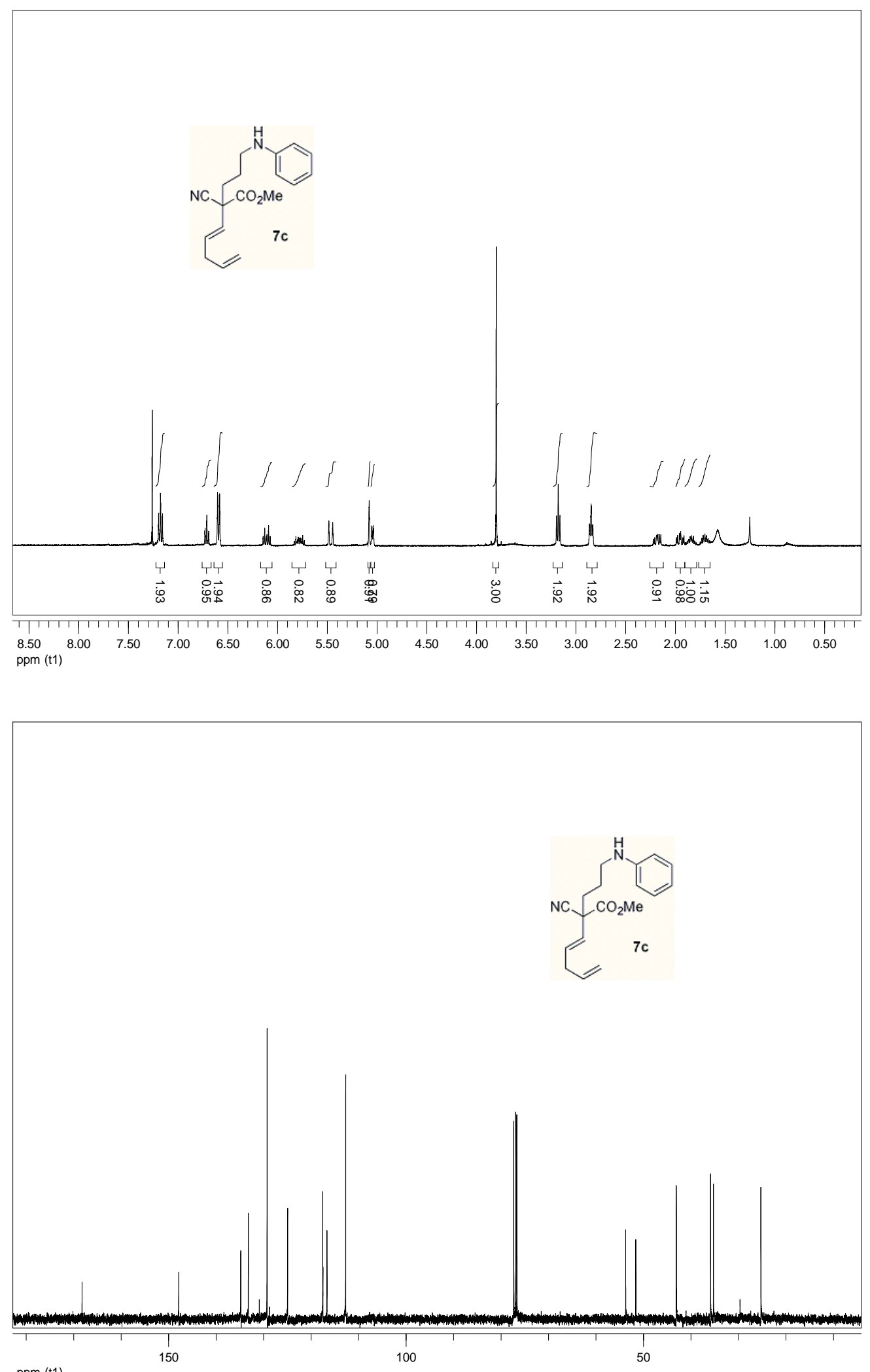

ppm (t1) 

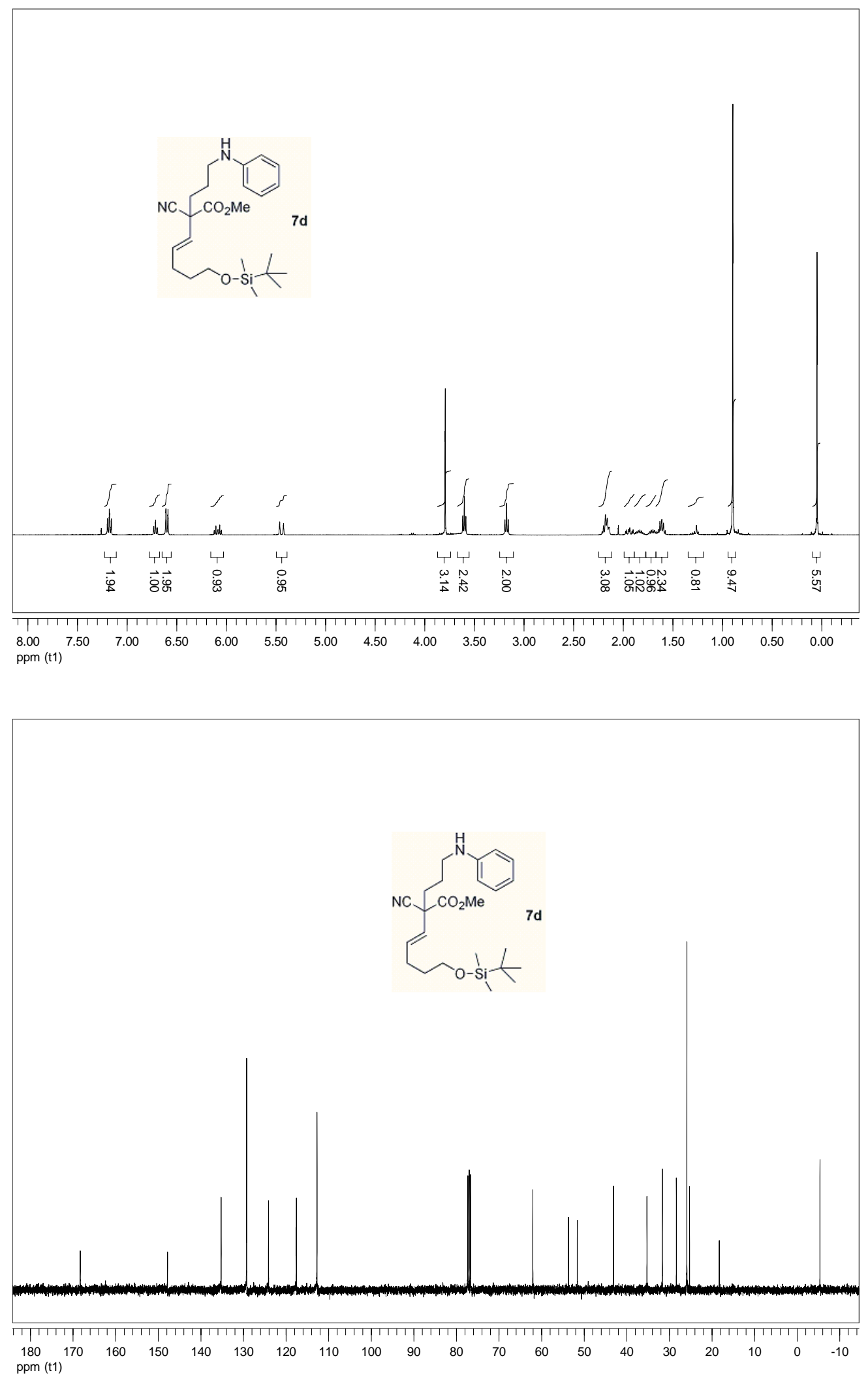

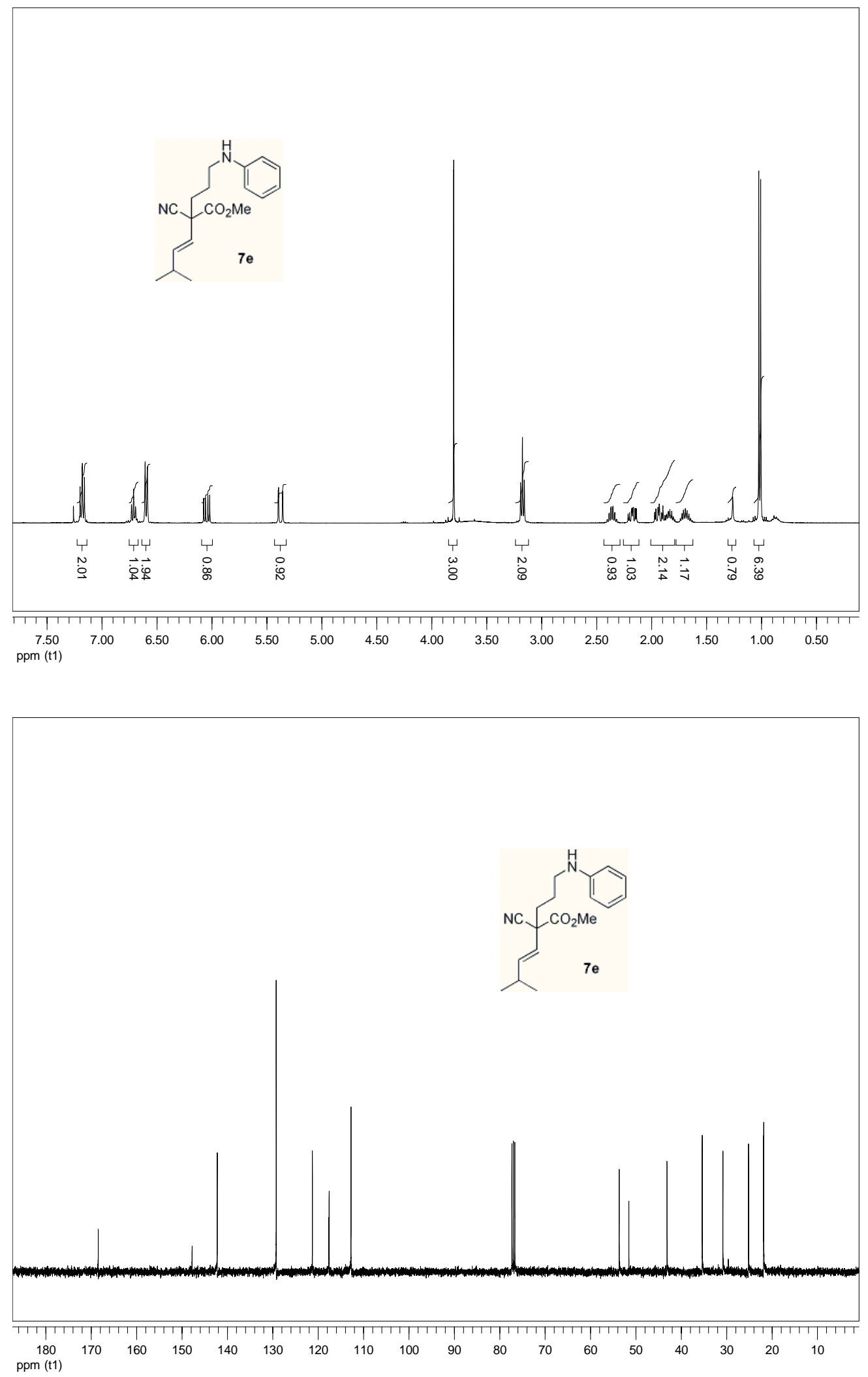


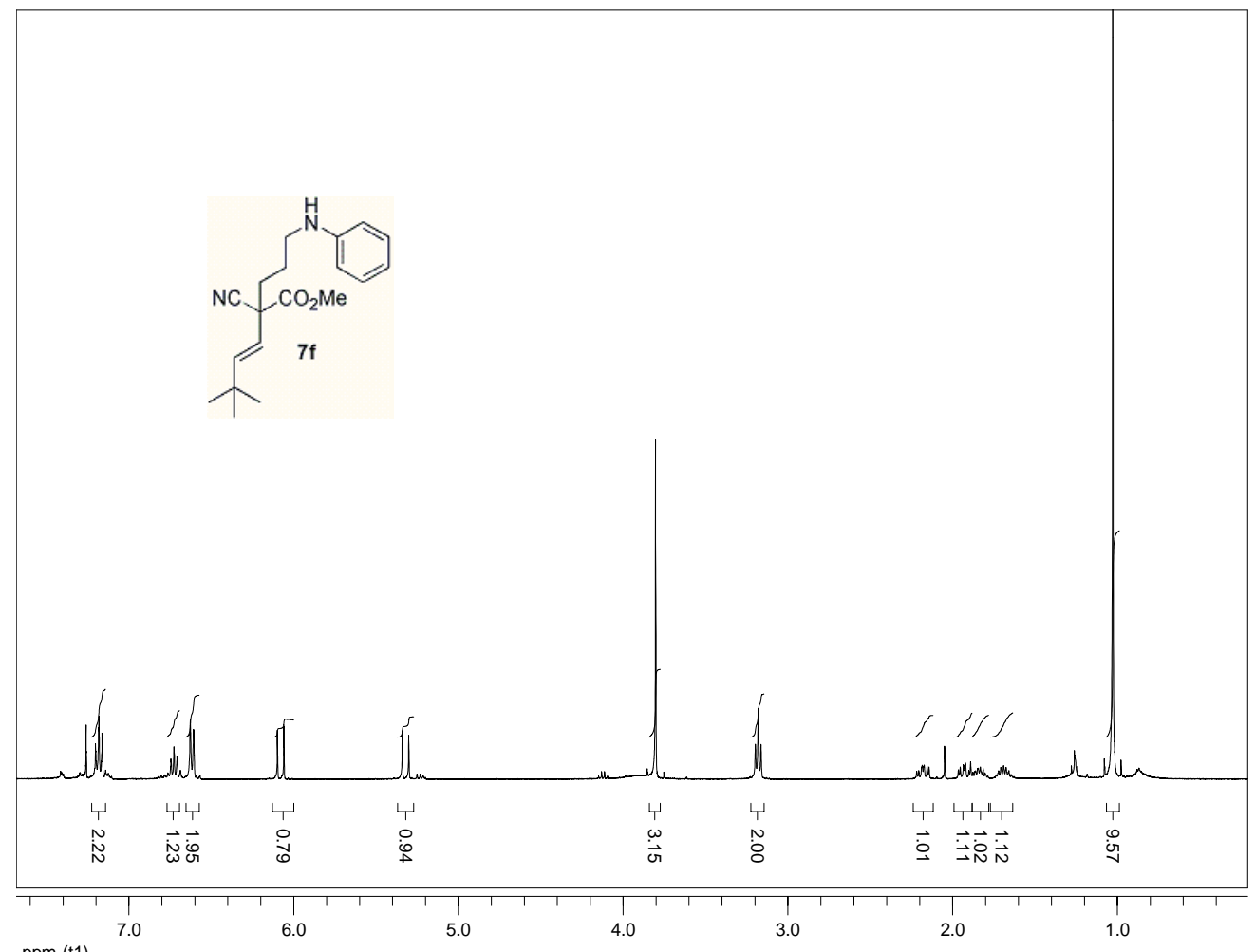

ppm (t1)

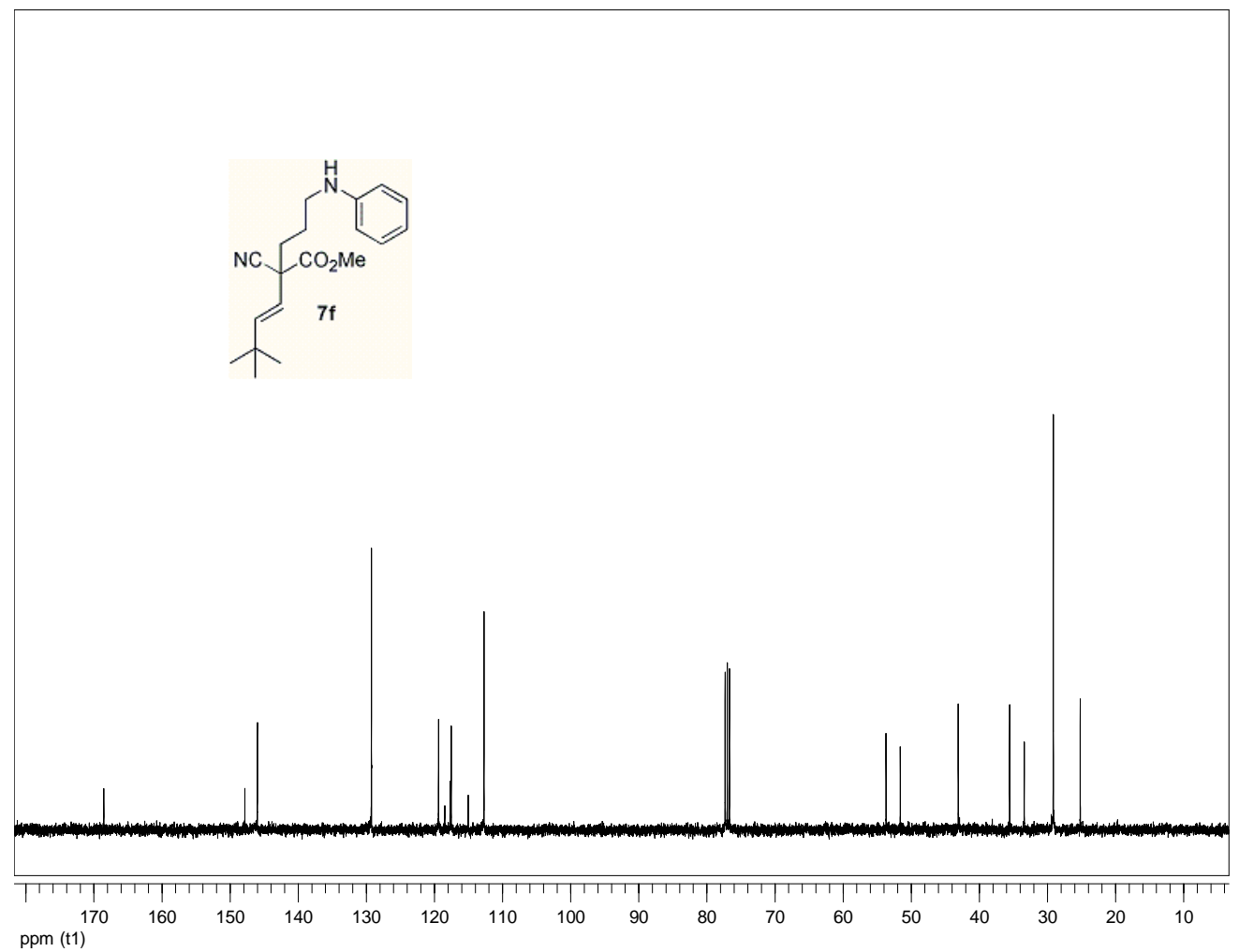



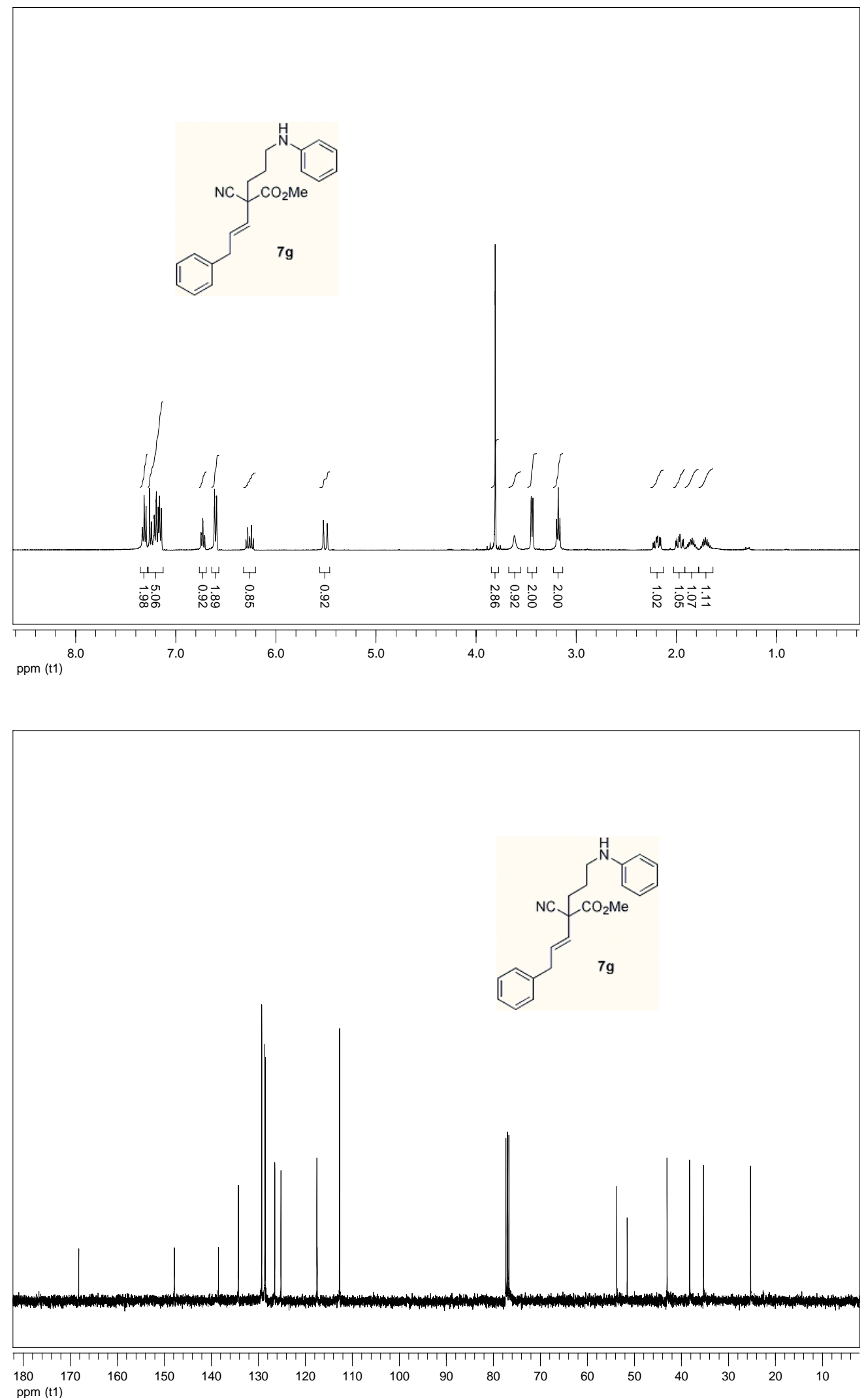

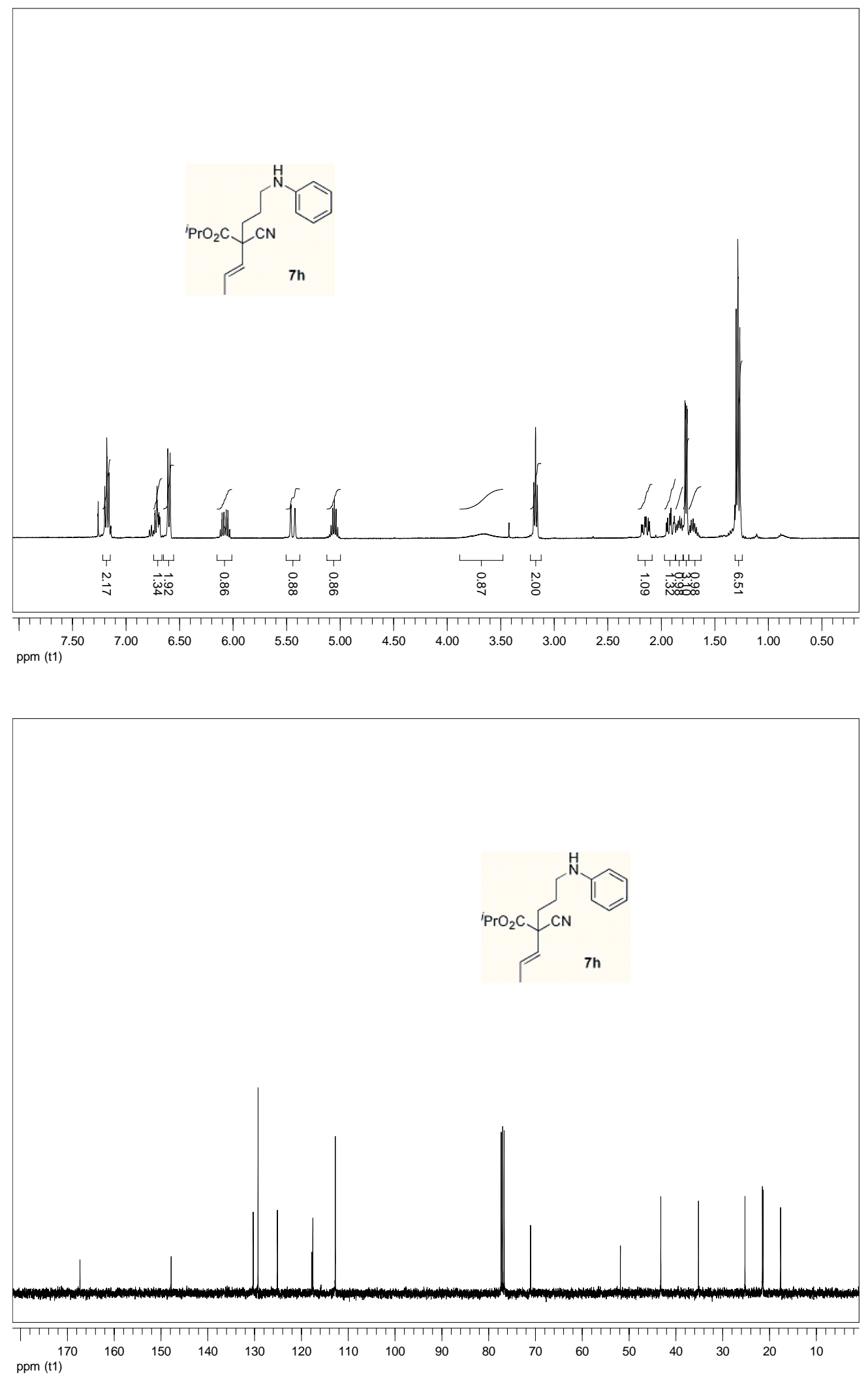

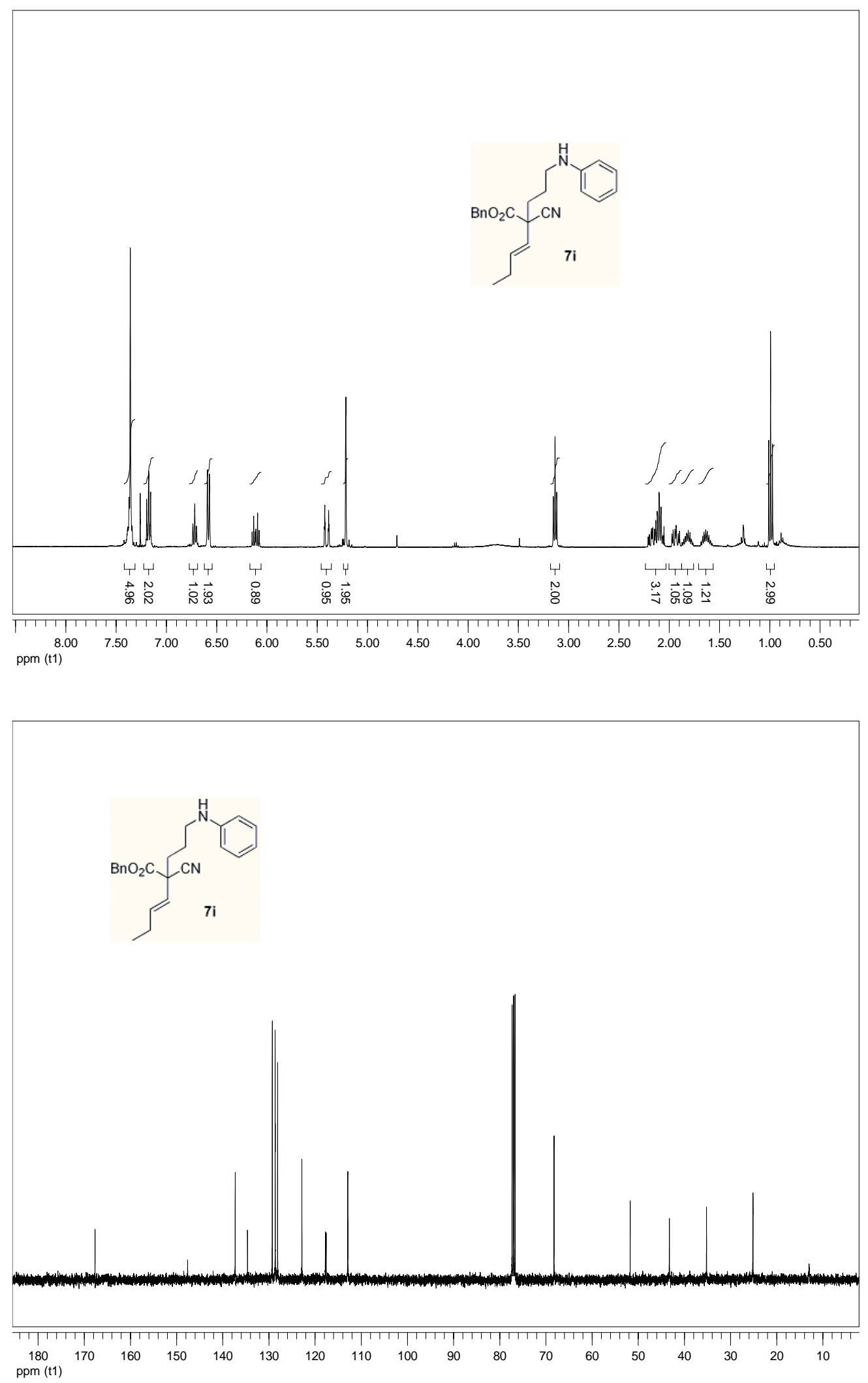

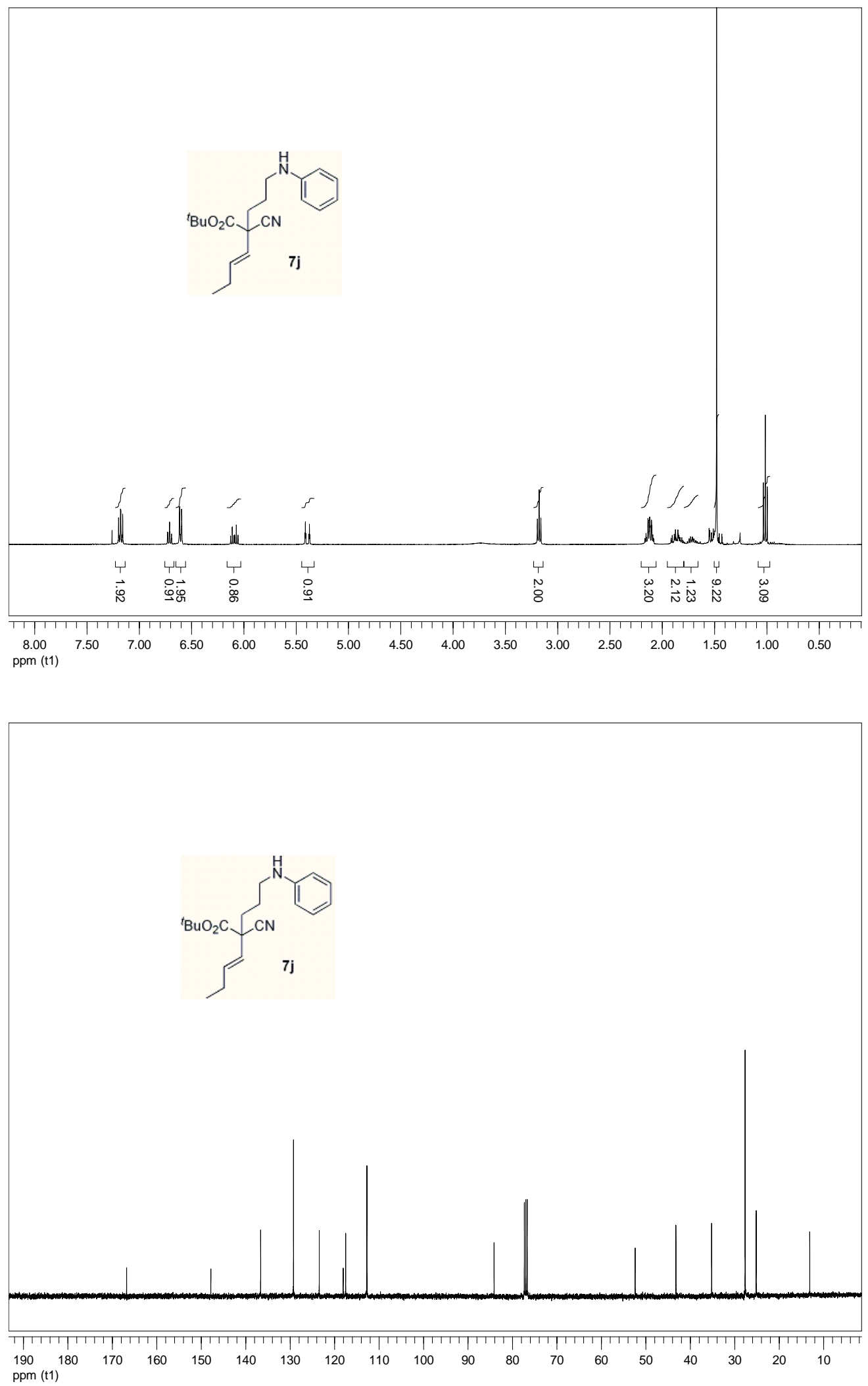

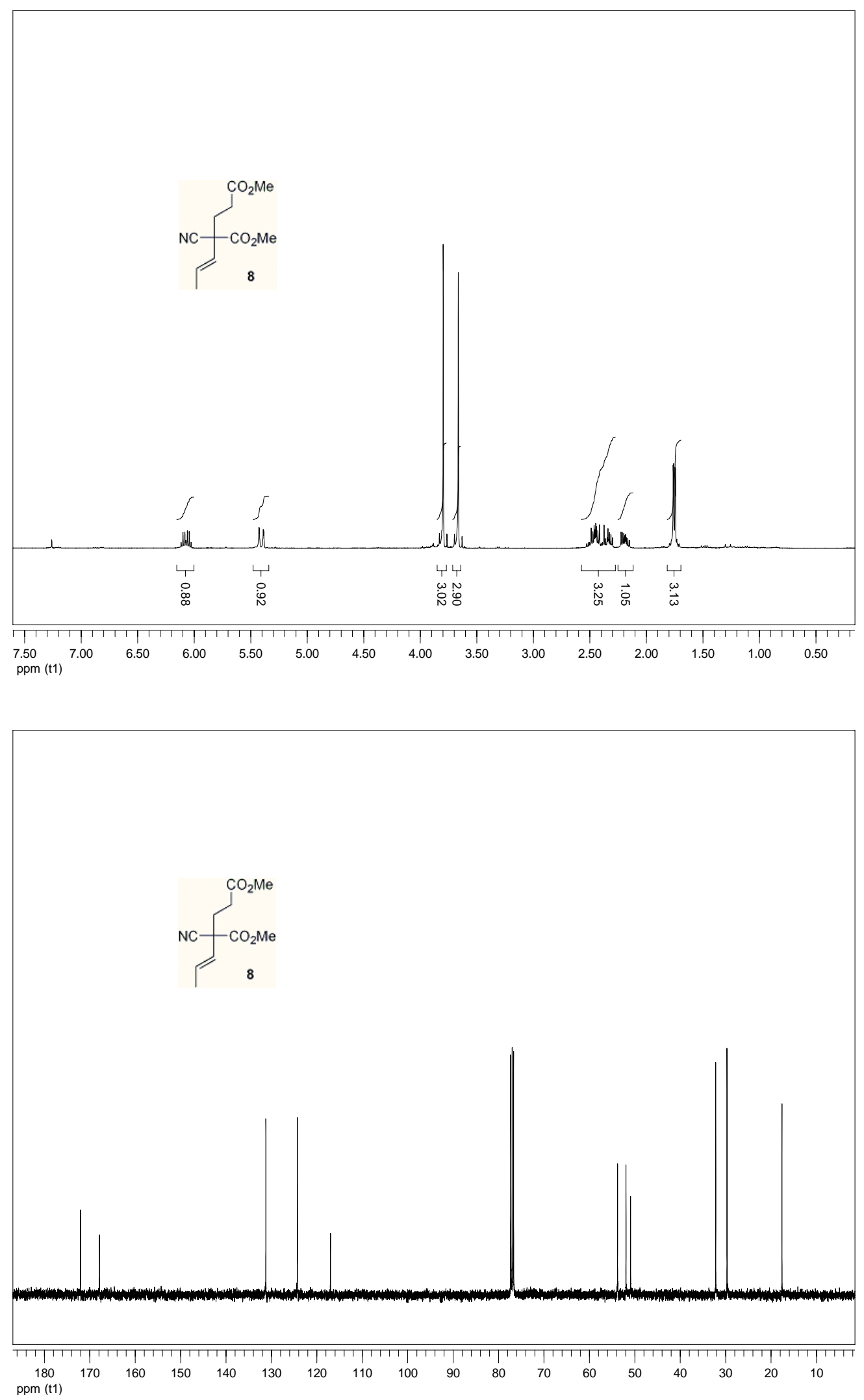

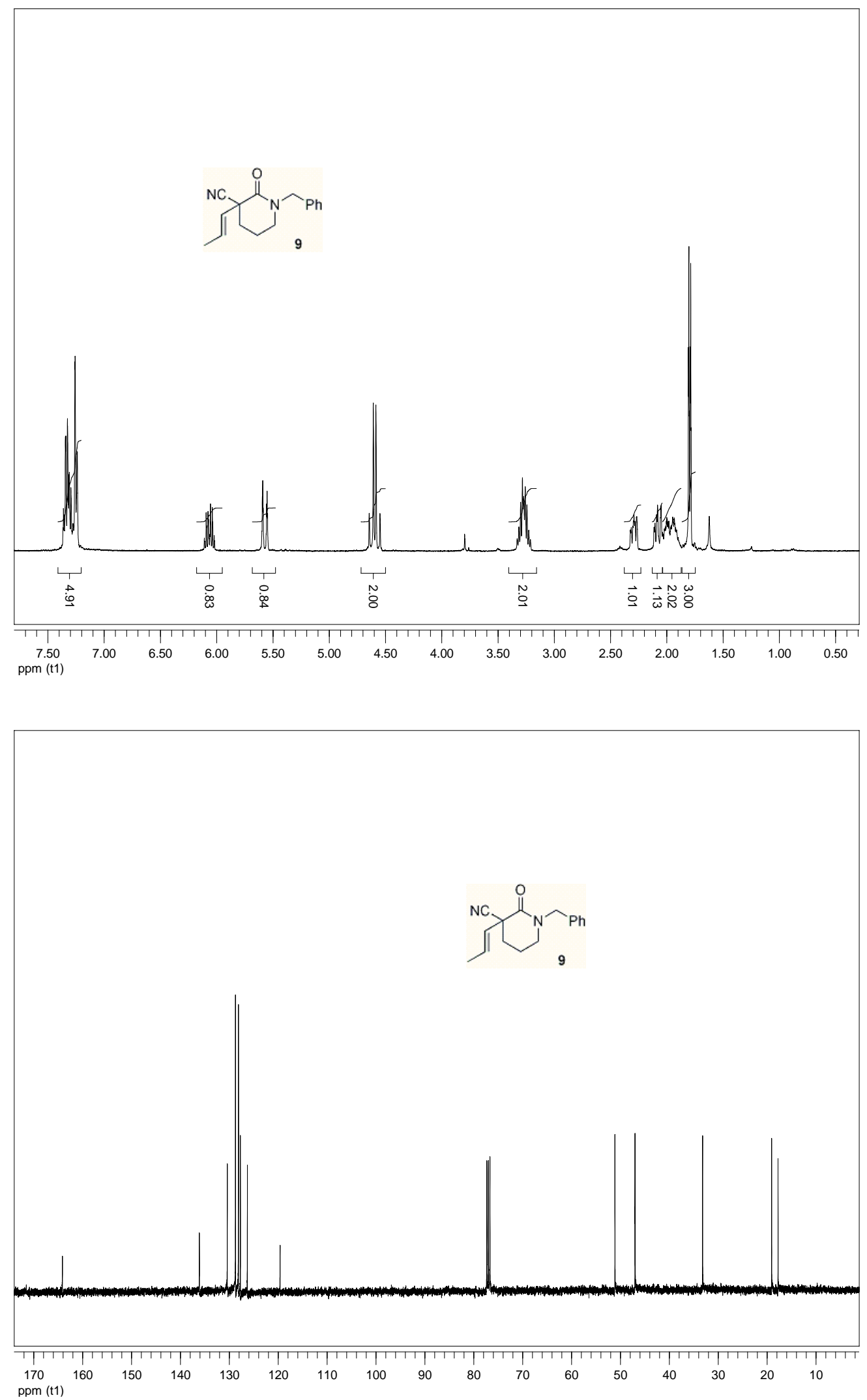

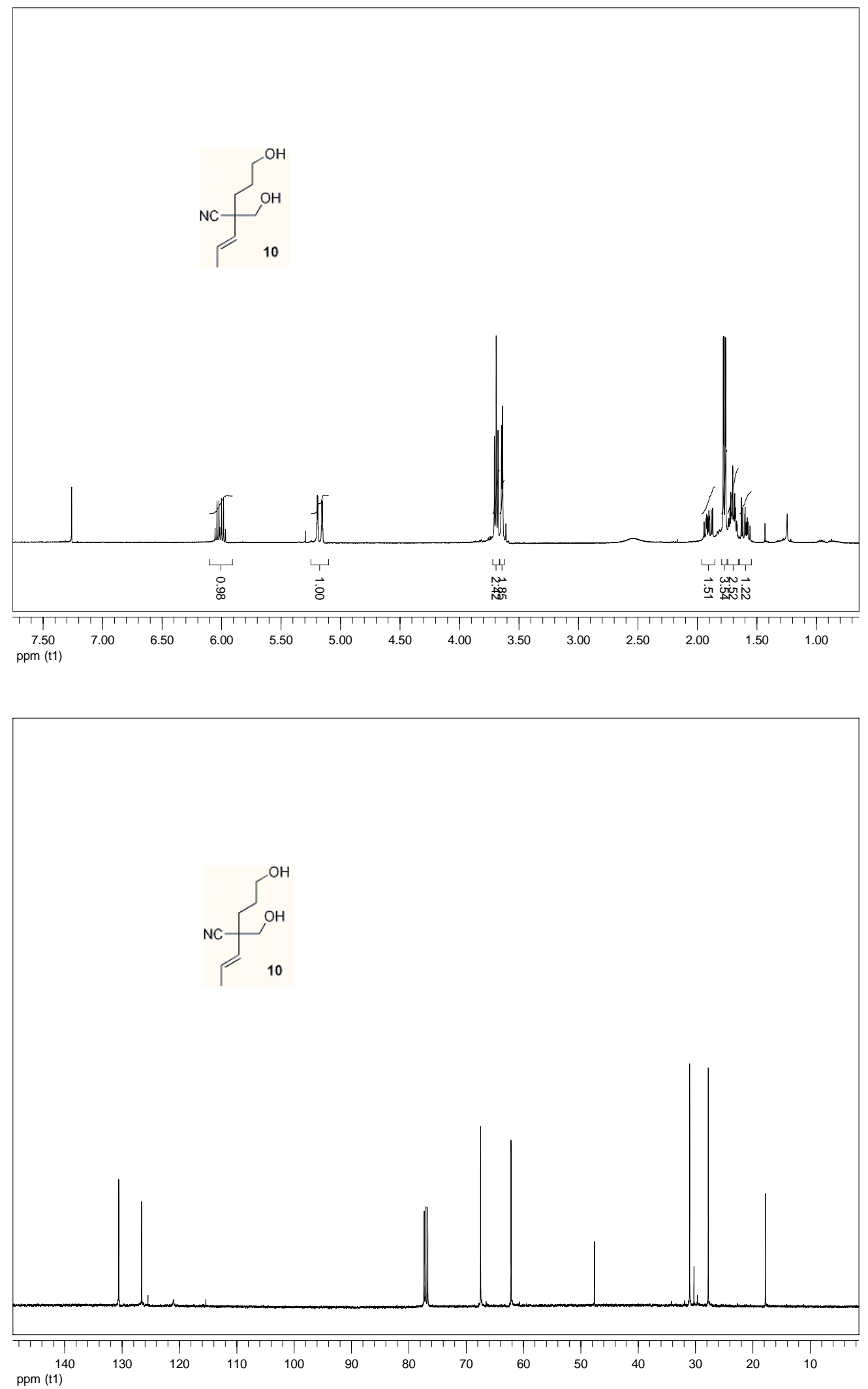

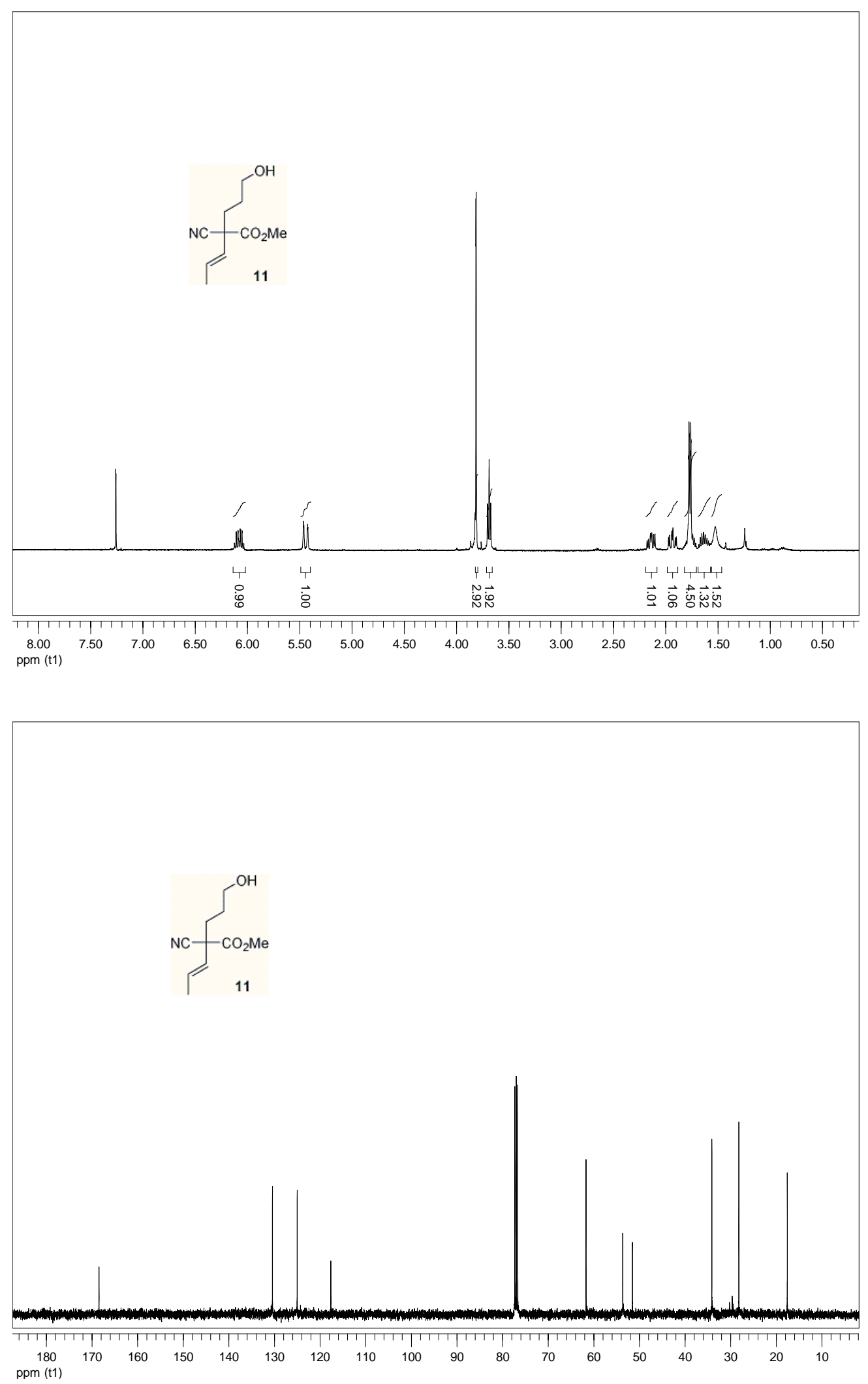

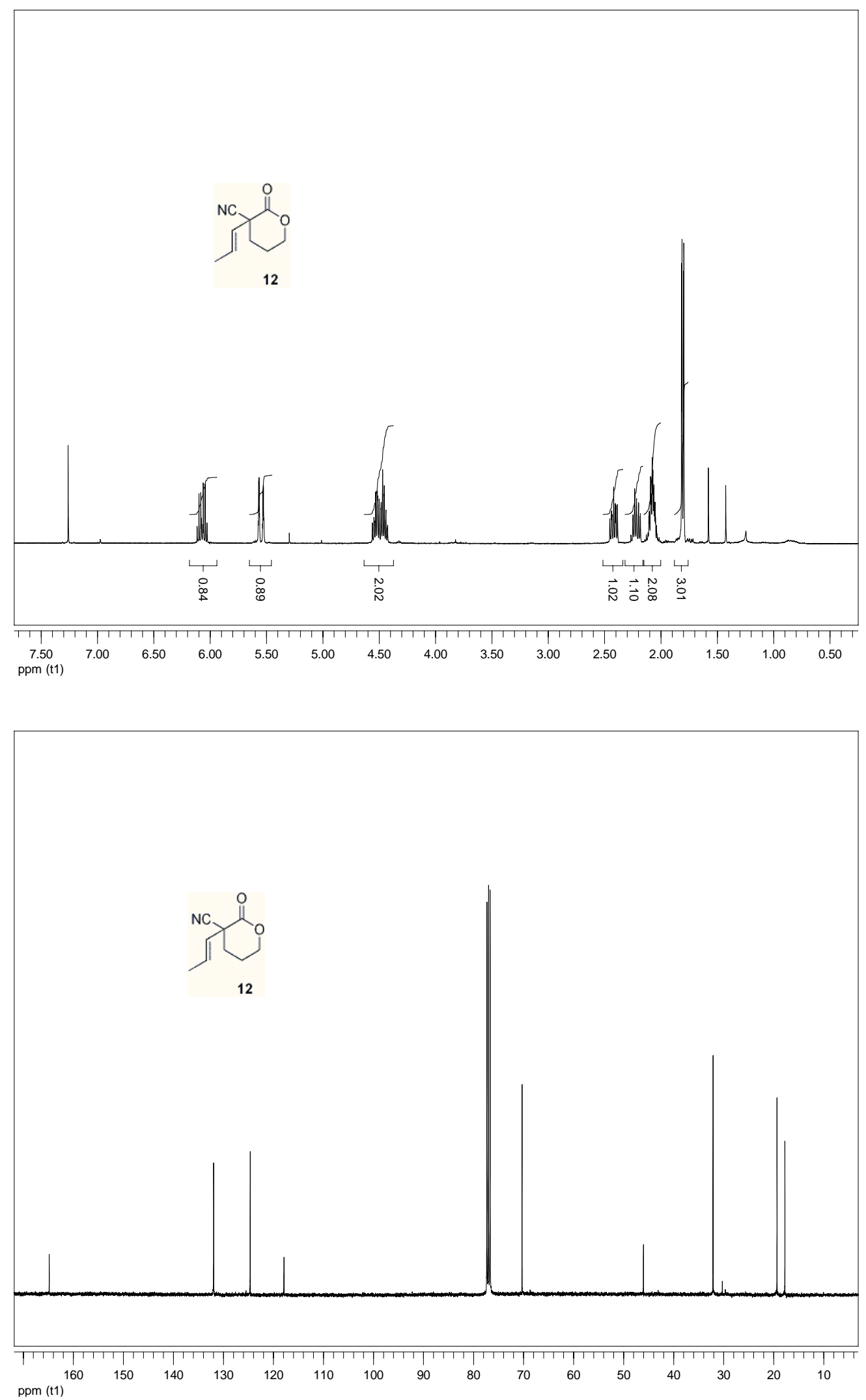


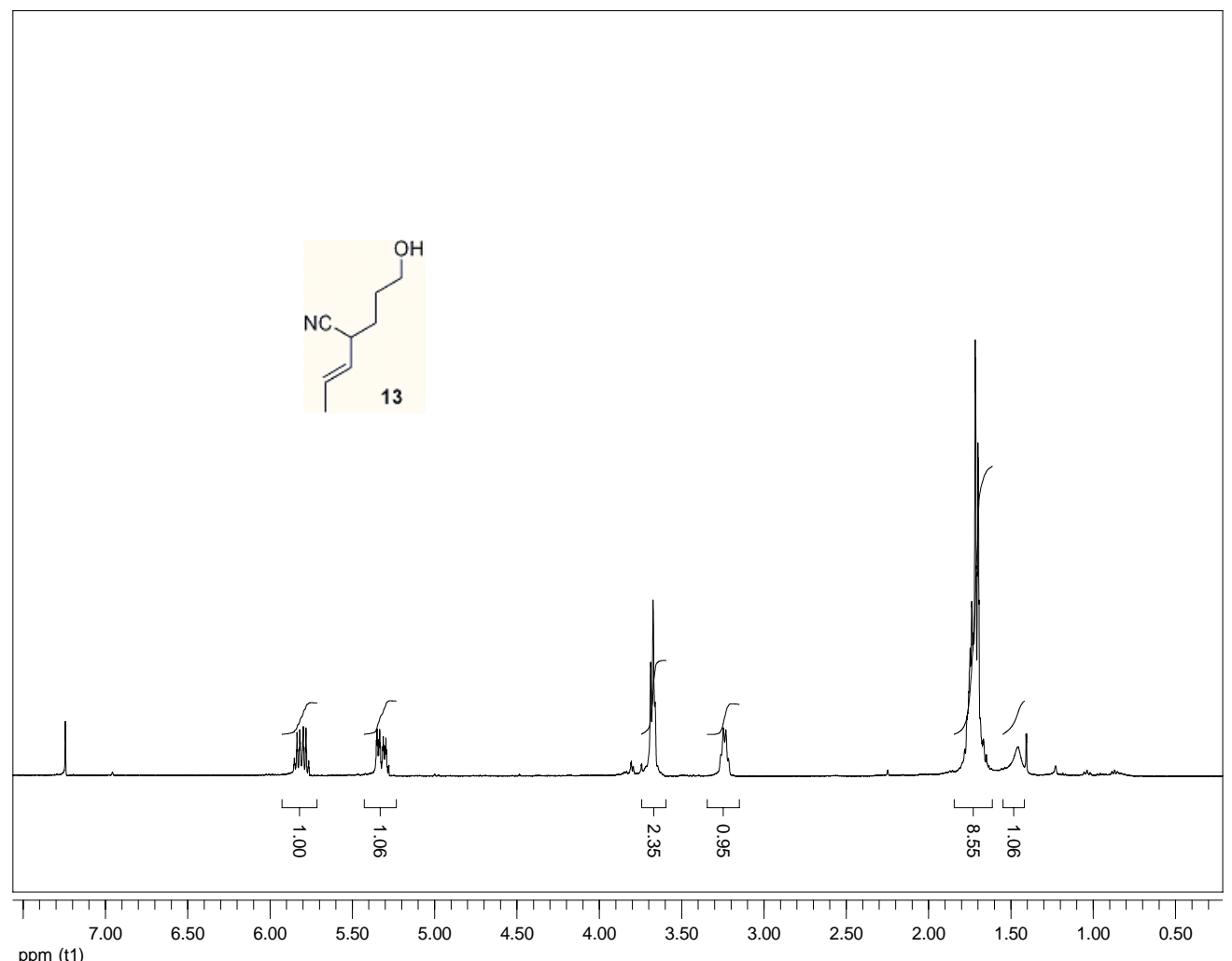
ppm (t1)

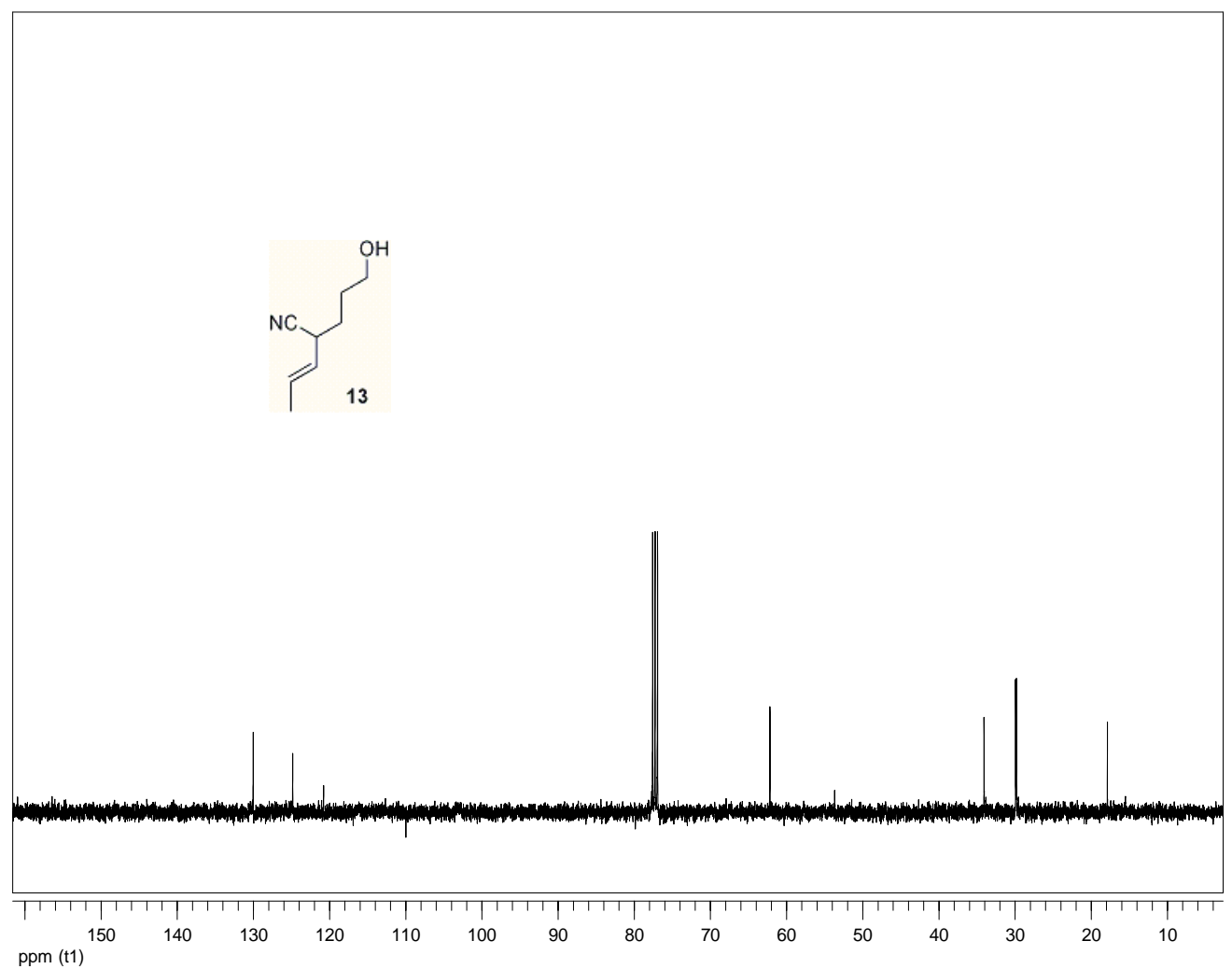




\section{References}

1. Busygin, I.; Toukoniitty, E.; Sillanpää, R.; Murzin, D. Y.; Leino, R. Eur. J. Org. Chem. 2005, 24, 2811.

2. Belle, D. D.; Tolvanen, A.; Lousasmaa, M. Tetrahedron 1996, 52, 11361.

3. Abdel-Magid, A. F.; Carson, K. G.; Harris, B. D.; Mryanoff, C. A.; Shah, R. D. J. Org. Chem. 1996, 61, 3849. 